\title{
Moduli stabilization in higher dimensional brane models
}

\author{
Antonino Flachi ${ }^{1 *}$, Jaume Garriga $^{1,2 \dagger}$, Oriol Pujolàs ${ }^{1 \ddagger}$ and Takahiro Tanaka ${ }^{3,4 \S}$ \\ ${ }^{1}$ IFAE, Campus UAB, 08193 Bellaterra (Barcelona), Spain \\ ${ }^{2}$ Departament de Física Fonamental and C.E.R. en Astrofísica, Física de Partícules i Cosmologia, \\ Universitat de Barcelona, Martí i Franquès 1, 08028 Barcelona, Spain \\ ${ }^{3}$ Institute of Cosmology, Department of Physics and Astronomy, Tufts University, Medford MA 02155, USA \\ ${ }^{4}$ Yukawa Institute for Theoretical Physics, Kyoto University, Kyoto 606-8502, Japan
}

(November 1, 2018)

\begin{abstract}
We consider a class of warped higher dimensional brane models with topology $\mathcal{M} \times \Sigma \times S^{1} / Z_{2}$, where $\Sigma$ is a $D_{2}$ dimensional manifold. Two branes of codimension one are embedded in such a bulk space-time and sit at the orbifold fixed points. We concentrate on the case where an exponential warp factor (depending on the distance along the orbifold) accompanies the Minkowski $\mathcal{M}$ and the internal space $\Sigma$ line elements. We evaluate the moduli effective potential induced by bulk scalar fields in these models, and we show that generically this can stabilize the size of the extra dimensions. As an application, we consider a scenario where supersymmetry is broken not far below the cutoff scale, and the hierarchy between the electroweak and the effective Planck scales is generated by a combination of redshift and large volume effects. The latter is efficient due to the shrinking of $\Sigma$ at the negative tension brane, where matter is placed. In this case, we find that the effective potential can stabilize the size of the extra dimensions (and the hierarchy) without fine tuning, provided that the internal space $\Sigma$ is flat.

Keywords: Extra dimensions; Brane models; Hierarchy stabilization
\end{abstract}

YITP-01-85

UAB-FT-539

\section{INTRODUCTION}

It is a widely accepted idea that our space-time might have more than four dimensions. This very old proposal $[1,2]$, revived in [3], is currently being considered in several contexts from particle physics to cosmology.

From a more fundamental perspective, string theory provides a strong motivation for considering higher dimensional scenarios. In a suggestive model [4,5], Hořava and Witten considered an 11-dimensional supergravity theory compactified on $\mathcal{M} \times \mathcal{C Y} \times S^{1} / Z_{2}$, where $\mathcal{M}$ represents four dimensional Minkowski space and $\mathcal{C Y}$ is a Calabi-Yau space, with the size of the Calabi-Yau much smaller than the orbifold one. In such a set-up, the fixed points of the $Z_{2}$ symmetry can be viewed as 10-dimensional boundaries which may accommodate a realistic gauge theory.

A closely related model has been considered by Randall and Sundrum [6], where two flat 3-branes of opposite tension are embedded in 5-dimensional Anti-de Sitter space. The extra dimension is compactified on a $Z_{2}$ orbifold, with the branes sitting at the fixed points. This results in a space-time with a non-factorisable geometry which, through a redshift effect, induces a four-dimensional effective scale on one of the branes which is much smaller than the 5 - dimensional cutoff scale (which is assumed to be Planckian). This geometric effect could explain the hierarchy between the Planck and the electroweak scales. The "radion" mode (which corresponds to the size of the extra dimension) plays a crucial role in this scenario, since its vacuum expectation value (VEV) determines the hierarchy. In the original RS model, the radion was massless at the classical level. This would cause an unacceptable modification of the resulting gravity on the brane [7], and therefore the radion must be stabilized by some mechanism [8]. Moreover, for a satisfactory solution to the hierarchy problem, such mechanism should give the radion a suitably large VEV without the need of introducing any fine tuning of parameters.

The problem of radion stabilization is not particular to the RS model, and arises generically in higher dimensional Kaluza-Klein theories, including the usual factorisable geometries based on direct products of Minkowski space with

\footnotetext{
*flachi@ifae.es

† garriga@ifae.es

${ }^{\ddagger}$ pujolas@ifae.es

§tanaka@yukawa.kyoto-u.ac.jp
} 
homogeneous manifolds. In that context, Candelas and Weinberg realized that the Casimir energy of matter fields or gravity could induce an effective potential for the radion at the quantum level, offering a natural mechanism for stabilizing the size of the extra dimensions [9]. Following this idea, a number of authors have investigated quantum stabilization in the context of brane models. In [10-16] the lowest order quantum corrections arising from bulk fields on the RS background have been calculated and their relevance to the radius stabilization problem has been investigated (see also [17-22] for discussions of the possible relevance of quantum effects in cosmological brane-world scenarios and [23] for finite temperature effects). Initially, such investigations were somewhat discouraging, since it was found [13] that in the RS model it is not possible to stabilize the extra dimension and simultaneously solve the hierarchy problem by means of the Casimir forces due to bulk gravitons or generic bulk scalars. However, it has recently been shown [24] that bulk gauge fields (or any of their supersymmetric relatives) can do the job. These fields induce logarithmic contributions to the radion effective potential which are sufficient to stabilize it, generating a large hierarchy of scales without fine-tuning. The logarithmic behaviour can be understood, in a $4 \mathrm{D}$ holographic description, as the running of gauge couplings with the infrared cut-off scale (which corresponds to the electroweak scale).

The RS model has opened up a very interesting framework for model building in particle physics, with possible cosmological implications. This scenario, however, is just the simplest possibility within a more general class of higher dimensional warped geometries which deserve fuller exploration, specially with regard to their possible embedding in string theory. In this connection, one expects that more general internal spaces and higher dimensionalities have to be considered, and in this paper we shall take a step in this direction. Our aim is twofold. On one hand, the consideration of more general spacetimes may provide interesting extensions of the RS mechanism for the geometric origin of the hierarchy. On the other, quantum effects in such scenarios can be qualitatively different, providing new ways of stabilizing the radion which do not necessarily rely on the peculiar behaviour of bulk gauge fields.

Generically, we expect that the behaviour of the effective potential for the "moduli" should be qualitatively different once we go beyond the RS scenario. This is indicated (even in five dimensions) by the study of generalized warped compactifications [14]. Aside from the non-local Casimir interaction between the branes which we mentioned above, local terms which are induced by quantum effects may stabilize the moduli when we consider warped brane worlds where the bulk is different from AdS. In the RS model both the branes and the bulk space-time are maximally symmetric and thus any possible counter-term amounts to a renormalization of the brane tensions. However, this is not true in general. An explicit example was worked out in detail in [14], where a class of 5-dimensional brane models with power law warp factors were investigated. In this case, the global symmetry which is responsible for the masslessness of the moduli at the classical level is shown to be anomalous. Thus, the effective potential develops terms which do not scale appropriately under the global symmetry and which therefore act as stabilizers for the moduli. Some of the 5 dimensional models considered in Ref. [14] can be obtained by dimensional reduction of $5+D_{2}$-dimensional models, and in this paper we shall focus on a class of higher dimensional models which includes those.

Specifically, we shall consider spaces with line element given by

$$
d s_{(D+1)}^{2}=e^{2 \sigma(y)} \eta_{\mu \nu} d x^{\mu} d x^{\nu}+R^{2} e^{2 \rho(y)} \gamma_{i j} d X^{i} d X^{j}+d y^{2} .
$$

The coordinates $x^{\mu}$ parametrize four dimensional Minkowski space $\mathcal{M}$ and the coordinates $X^{i}$ cover a $D_{2}-$ dimensional compact internal manifold $\Sigma$. We locate two $D \equiv\left(4+D_{2}\right)$-dimensional branes at the fixed points of the orbifolded dimension labelled by $y$. Such a metric is found as a solution of a $(D+1)$-dimensional system of gravity plus certain 'matter' fields. Depending on the field content, different warpings can arise. For instance, Gregory showed in [25] that a six dimensional global string solution exists for negative cosmological constant, with $\sigma(y)=-k|y|$ and $\rho(y)=$ constant. Gherghetta and Shaposhnikov [26] constructed the metric solution of a six dimensional local string-like defect with $\sigma(y)=\rho(y)=-k|y|$. Generalizations of this models with more extra dimensions with $\Sigma=S^{D_{2}}$ were considered in in $[27,28]$ using bulk scalar fields with a hedgehog configuration.

The authors of [29] include Yang-Mills (YM) fields with appropriately chosen gauge group, instead. They find a series of solutions classified in terms of the Ricci flatness of the manifold $\Sigma$ : when the internal space is Ricci flat (for example a $D_{2}$-dimensional torus or a Calabi-Yau space), one obtains warp factors which can generically be expressed as sums of exponentials if there is no YM flux; in particular, when the bulk cosmological constant is negative, a specially simple solution with both warp factors equal to the RS one exists (including the case of higher dimensional $A d S$ space). Turning on some YM flux can relax the condition of the Ricci flatness of the internal manifold. In this case, they find a solution where along the Minkowski direction the warp is $\grave{a}$ la RS, whereas along the curved manifold it is constant. This gives the interesting combination of a higher dimensional theory which is a hybrid between an ordinary Kaluza-Klein theory and the RS model. In passing, we note that the phenomenology of such a scenario has been recently considered in [30,31].

In the present paper we start considering quantum effects from bulk fields quantized on such space-times beginning with the case when the two warp factors are of the RS type. The computation is similar in some respect to the RS 
one with some additional technical complications. Moreover we allow a more general setting than the one presented in [29] in that we consider solutions with internal manifolds $\Sigma$ which are not Ricci flat. In section II we describe how to construct these types of models (for the case of Ricci flat internal manifolds we refer the reader to reference [29]) and subsequently discuss the moduli approximation in this type of space-times.

The Kaluza-Klein reduction of the bulk field is considered in section III, where it is shown that, when branes have a non trivial topology, the physical mass depends also on the eigenvalues of the Klein-Gordon operator on the internal manifold. The one-loop effective action is evaluated in section IV and the result is simply expressed in terms of heat-kernel coefficients. This is done using the Mittag-Leffler expansion of the generalized $\zeta$ function. The result is regulated by using dimensional regularization and appropriately renormalized in section $\mathrm{V}$.

In section VI, we propose a scenario where supersymmetry is broken just below the cutoff scale and the hierarchy is generated by a combination of redshift and large volume effects. The possibility of stabilizing the moduli (and the hierarchy) with the quantum effective potential is discussed in section VII for the different cases of interest and it is shown that such a stabilization can in fact take place without fine tuning. The conclusions are left to the last section.

The paper is equipped with an appendix in which we obtain the Mittag-Leffler expansion for a positive semidefinite operator (This last result does not claim any originality and can possibly be found elsewhere). Appendix B contains the details of the dimensional reduction of the gravitational part of the action.

\section{SETUP}

We are interested in the quantum effective action arising from a quantized bulk scalar field on the background space-time specified by the metric (1). We consider two branes of codimension one with the topology of $\mathcal{M}^{4} \times \Sigma$, where the manifold $\Sigma$ is taken to be Einstein and compact. The branes sit at the orbifold fixed points and the $Z_{2}$ symmetry is imposed on the solutions.

As we have already mentioned in the introduction, such solutions have been obtained in [29] for Ricci flat internal manifolds. However, as also shown in [27,28], more general solutions with $\Sigma=S^{D_{2}}$ can be found by introducing additional matter content coming from a scalar field with a hedgehog configuration. In the next Section, we show how to obtain such a solution.

\section{A. Model}

The specific space-time that we consider in this paper corresponds to the case when the two warp factors are equal and exponential, $\sigma(y)=\rho(y)=-k|y|$. The model consists of an $G$ invariant non-linear sigma model parametrized by a set of bulk scalar fields $\phi^{a}$ together with a standard bulk gravity sector, and two boundary-branes. This is described by the action

$$
\begin{aligned}
S & =\int d^{D+1} x \sqrt{g_{(D+1)}}\left\{M^{D-1} \mathcal{R}_{(D+1)}-\Lambda-\partial_{M} \phi^{a \dagger} \partial^{M} \phi^{a}-\lambda\left(\phi^{a \dagger} \phi^{a}-v^{2}\right)\right\} \\
& -\int d^{D} x \sqrt{g_{(D)+}} \tau_{+}-\int d^{D} x \sqrt{g_{(D)-}} \tau_{-} .
\end{aligned}
$$

Our notation the following. The higher dimensional bulk indices are $M, N, \ldots$ and run over $\mu, i, y ;$ the $\left(4+D_{2}\right) \equiv D$ dimensional brane indices are $A, B, \ldots$ and run over $\mu, i ; g_{M N}^{(D+1)}$ is the bulk metric and $g_{A B}^{(D) \pm}$ are the induced metrics on the branes. Finally, $\tau_{ \pm}$are the brane tensions, and $M$ is the higher dimensional fundamental Planck mass.

Let us look more closely at the structure of the scalar fields. The equation of motion for the scalars can be written as usual:

$$
\square \phi^{a}=-\lambda \phi^{a}
$$

The role of non-dynamical auxiliary field $\lambda$ is to impose the constraint

$$
\phi^{a \dagger} \phi^{a}-v^{2}=0
$$

Differentiating this constraint twice, we can rewrite Eq. (3) as follows:

$$
\square \phi^{a}=-\left(\frac{\partial_{M} \phi^{b} \partial^{M} \phi^{b \dagger}}{v^{2}}\right) \phi^{a} .
$$


The previous equation allows hedgehog solutions for $\phi^{a}$ for suitable choices of the group $G$. Moreover, they have a constant profile along the orbifold and satisfy

$$
\Delta_{\gamma} \phi^{a}=-L^{2} \phi^{a}, \quad \text { and } \quad \partial_{M} \phi^{a \dagger} \partial^{M} \phi^{a}=e^{-2 \rho} \frac{L^{2} v^{2}}{R^{2}}
$$

where $L$ is a 'winding number', and $\Delta_{\gamma}$ is the laplacian obtained from $\gamma_{i j}$.

The Einstein equations for such hedgehog configurations have been studied in [26-28], where solutions of the type (1) with $\sigma(y)=\rho(y)=-k y$ have been found, with

$$
k=\sqrt{-4 M^{1-D} \Lambda /(D-1) D},
$$

where $\Lambda<0$. In order to obtain the space-time described previously we take two copies of a slice of this $D+1$ dimensional space comprised between $y_{+}$and $y_{-}$, corresponding to the brane locations. The two copies are glued together there. Along with the the identification $y-y_{ \pm} \rightarrow 2 y_{ \pm}-y$, this gives the topology of an $S^{1} / Z_{2}$ orbifold in the $y$ direction.

In order for this to be a solution of our model (2), the brane tensions have to satisfy

$$
\tau_{ \pm}= \pm 4 \sqrt{-(D-1) M^{D-1} \Lambda / D}= \pm 4(D-1) M^{D-1} k,
$$

as a result of the junction conditions at the branes. Besides (6), the Einstein equations in the bulk relate the hedgehog parameters to the curvature of the internal manifold $\Sigma$ as

$$
v^{2}=\frac{2 D_{2} C}{L^{2}} M^{D-1}
$$

Here, since $\Sigma$ is homogeneous, the dimensionless constant $C$ is defined through $\mathcal{R}_{i j}^{(\gamma)}=C \gamma_{i j}$, and $\mathcal{R}_{i j}^{(\gamma)}$ is the Ricci tensor computed out of $\gamma_{i j}$.

Associated to the sigma model scalars a number of Nambu Goldstone modes will be present. However, we shall assume that these couple to matter only through gravity, so that their effects are negligible.

\section{B. Moduli}

One interesting feature of this ansatz is that the parameter $R$, describing the volume of $\Sigma$, does not appear in the equations of motion even in the case of a curved internal space. Moreover, the positions of both branes are free at the classical level. They correspond to flat directions in the action and thus are the relevant degrees of freedom at low energies. In the moduli approximation, which we shall follow here, they are promoted to four dimensional scalar fields.

One crucial difference of these solutions with respect to the RS model is that they are not homogeneous along the orbifold, even in the case when $\Sigma$ is a torus ${ }^{1}$. This is due to the compactness of $\Sigma$. In contrast with the RS model, the positions of both branes are physically meaningful.

However, it is clear that a scaling of $R$ is equivalent to a shift in the positions of the branes $y_{ \pm}$. Therefore, they are not independent. Rather, only two moduli are needed. Since we will use several combinations of the moduli along the paper, we summarize them briefly now: $a_{ \pm} \equiv e^{-k y_{ \pm}}$, the physical radii of $\Sigma$ at the branes $R_{ \pm}=a_{ \pm} R$, the corresponding dimensionless values $r_{ \pm}=a_{ \pm} k R$, and $a \equiv e^{-k\left(y_{-}-y_{+}\right)}=a_{-} / a_{+}$.

In addition to the moduli, the massless sector also contains the graviton zero mode. To take it into account, we perturb the background solution (1) as follows

$$
d s^{2}=d y^{2}+e^{2 \sigma(y)}\left[\tilde{g}_{\mu \nu}(x) d x^{\mu} d x^{\nu}+R^{2} \gamma_{i j} d X^{i} d X^{j}\right] .
$$

Substituting this metric back into the action (2) we obtain the kinetic term for $\tilde{g}$ coming from the bulk part (see [14]). The kinetic terms for the moduli $y_{ \pm}$come from the boundary terms. A computation analogous to that in [14] gives

$$
S_{(4)}=-m_{P}^{2} \int d^{4} x \sqrt{-\tilde{g}}\left\{\left[\varphi_{+}^{2}-\varphi_{-}^{2}\right] \tilde{\mathcal{R}}-4 \frac{D-1}{D-2}\left[\left(\tilde{\partial} \varphi_{+}\right)^{2}-\left(\tilde{\partial} \varphi_{-}\right)^{2}\right]\right\}
$$

\footnotetext{
${ }^{1}$ In this case, the solution corresponds to a toroidal compactification of a higher dimensional $A d S$ space.
} 
where $\varphi_{ \pm}^{2}=a_{ \pm}^{D-2}=e^{-(D-2) k y_{ \pm}}$, and the effective four dimensional Planck mass is given by

$$
m_{P}^{2}=\frac{2}{D-2} v_{\Sigma} R^{D_{2}} M^{D-1} / k, \quad \text { with } \quad v_{\Sigma}=\int_{\Sigma} \sqrt{\gamma} d^{D_{2}} X .
$$

We note that the moduli $\varphi_{ \pm}$are Brans-Dicke (BD) fields and in the frame defined by $\tilde{g}_{\mu \nu}$, the kinetic term for $\varphi_{+}$ has the wrong sign. Introducing the new variables $\varphi$ and $\psi[14,32]$,

$$
\varphi_{+}=\varphi \cosh \psi \quad \text { and } \quad \varphi_{-}=\varphi \sinh \psi
$$

the Einstein frame is given by $\hat{g}_{\mu \nu}=\varphi^{2} \tilde{g}_{\mu \nu}$. In this frame the action (10) takes the form

$$
S_{(4)}=-m_{P}^{2} \int d^{4} x \sqrt{-\hat{g}}\left\{\hat{\mathcal{R}}+2 \frac{D_{2}}{D_{2}+2}(\hat{\partial} \ln \varphi)^{2}+4 \frac{D_{2}+3}{D_{2}+2}(\hat{\partial} \psi)^{2}\right\}
$$

and now the kinetic terms are both positive definite. Moreover, we note that the modulus $\varphi$ decouples in the limit $D_{2} \rightarrow 0$, as expected, since this case corresponds to the usual RS model, where only one modulus is present.

We are assuming that the ( $D$ dimensional) matter fields $\chi_{ \pm}^{(D)}$ are localized on each brane and so they couple universally to the corresponding induced metrics $g_{A B}^{(D) \pm}$ (recall $A, B, \cdots=\mu, i$ )

$$
S^{\text {matt }}=\sum_{ \pm} \int d^{D} x \sqrt{-g^{(D) \pm}} \mathcal{L}^{ \pm}\left(\chi_{(D)}^{ \pm}, g_{A B}^{(D) \pm}\right)=\int d^{4} x \sum_{ \pm} \sqrt{-g_{ \pm}} a_{ \pm}^{D_{2}} \mathcal{L}^{ \pm}\left(\chi^{ \pm}, g_{\mu \nu}^{ \pm}\right)
$$

Here, we have kept the $\Sigma$-zero modes only, and integrated out the $X$ dependence, the $\Sigma$ volume factor has been absorbed by the four dimensional matter fields $\chi$ and couplings, and the four dimensional induced metrics are the $(\mu, \nu)$ components of the $D$ dimensional ones $g_{\mu \nu}^{ \pm}=g_{\mu \nu}^{(D) \pm}$.

A repeated use of the chain rule leads to the interaction of the moduli with matter given by

$$
S^{\text {mod-matt }}=\int d^{4} x \sqrt{-\hat{g}}\left\{-\frac{D_{2}}{D_{2}+2} \sum_{ \pm}\left[\hat{T}_{ \pm}-2 \hat{\mathcal{L}}_{ \pm}\right] \delta \ln \varphi+\frac{2}{D_{2}+2} \sum_{ \pm} a^{ \pm\left(D_{2}+2\right) / 2}\left[\hat{T}_{ \pm}+D_{2} \hat{\mathcal{L}}_{ \pm}\right] \delta \psi\right\},
$$

where $\hat{T}_{ \pm}$and $\hat{\mathcal{L}}_{ \pm}$are defined according to $\sqrt{-g_{ \pm}} T_{ \pm}=\sqrt{-\hat{g}} \hat{T}_{ \pm}$, and $\sqrt{-g_{ \pm}} a_{ \pm}^{D_{2}} \mathcal{L}_{ \pm}=\sqrt{-\hat{g}} \hat{\mathcal{L}}_{ \pm}$. The coupling of the moduli to the Lagrangian is entirely due to the dimensions along $\Sigma$ being warped, and is a generic prediction of models with a nontrivial warp factor for the extra dimensions. In fact, the 'radion' modulus $\Psi$ is coupled to matter through $\left(\hat{T}+D_{2} \hat{\mathcal{L}}\right)_{ \pm}$, which coincides with the trace of the $D$ dimensional energy momentum tensor. Moreover, this shows that the the modulus $\varphi$ decouples from matter in the RS limit $D_{2} \rightarrow 0$, as it should.

Defining the canonical fields

$$
\Phi=2 \sqrt{\frac{D_{2}}{D_{2}+2}} m_{P} \delta \ln \varphi, \quad \text { and } \quad \Psi=2 \sqrt{2 \frac{D_{2}+3}{D_{2}+2}} m_{P} \delta \psi,
$$

we obtain the equations of motion for the moduli

$$
\begin{aligned}
& \square \Phi=\frac{1}{2} \sqrt{\frac{D_{2}}{D_{2}+2}} \frac{1}{m_{P}}\left[\hat{T}_{+}-2 \hat{\mathcal{L}}_{+}+\hat{T}_{-}-2 \hat{\mathcal{L}}_{-}\right] \\
& \square \Psi=-\frac{1}{\sqrt{2\left(D_{2}+3\right)\left(D_{2}+2\right)} m_{P}}\left[a^{\left(D_{2}+2\right) / 2}\left(\hat{T}_{+}+D_{2} \hat{\mathcal{L}}_{+}\right)+a^{-\left(D_{2}+2\right) / 2}\left(\hat{T}_{-}+D_{2} \hat{\mathcal{L}}_{-}\right)\right] .
\end{aligned}
$$

As we explain in Sec. VI, we are interested in the case of $a \ll 1$ in order to have a substantial redshift effect arising from the warp factors. Unless otherwise stated, we shall set $\left\langle a_{+}\right\rangle=1$, so that, with a good accuracy, $a_{-} \simeq a$, $\varphi \simeq \varphi_{+} \simeq 1$ and $\psi \simeq \varphi_{-} \ll 1$

Thus, from (15) we can read off the couplings to the two types of matter: ${ }^{2} \Phi$ couples to the matter at either brane $\chi_{ \pm}$, with a strength $\sim 1 / m_{P}$. As for $\Psi$, the coupling to $\chi_{-}$is quite large, of order $a^{-\left(D_{2}+2\right) / 2} / m_{P}$, and to $\chi_{+}$is even smaller than Planckian, $\sim a^{\left(D_{2}+2\right) / 2} / m_{P}$.

\footnotetext{
${ }^{2}$ In the rest of this article, we will consider only matter located on the negative tension brane. Here we just consider other possible forms of matter at $y=y_{+}$for the sake of generality.
} 


\section{KALUZA-KLEIN REDUCTION}

Before the evaluation of the one-loop effective action, which we do in Section IV, we turn now to the reduction in KK modes of a bulk scalar field living in the space-time described in the previous section.

The idea is very simple: by performing a Kaluza-Klein reduction of the higher dimensional scalar field theory from $D+1$ (with $D=D_{1}+D_{2}$ ) down to $D_{1}$ dimensions, we obtain an equivalent lower dimensional theory consisting of an infinite number of massive Kaluza-Klein modes. Specifically, the Kaluza-Klein reduction is performed by expanding the higher dimensional scalar field in terms of a complete and orthogonal set of modes and then integrating out the dependence on the extra dimensions. The masses turn out to be quantized according to some eigenvalue problem and depend on the details of the space-time, the nature of the internal manifold and on the bulk (higher dimensional) scalar field. The one-loop effective action can then be evaluated by re-summing the contribution of each one of the modes.

Typically in Kaluza-Klein theory the mass eigenvalues are found explicitly and the subsequent evaluation of the sum over the modes does not present particular difficulties. However, in the case of warped space-times the main difference is that the orbifold nature of the extra dimension complicates the mass eigenvalues, which are expressed in terms of a transcendental equation and thus cannot be found explicitly.

In the present section we will carry out the first step of the computation, namely the Kaluza-Klein reduction of the bulk scalar field. We will consider the most general case of a massive non-minimally coupled scalar field and assume that $\Sigma$, a compact manifold.

The bulk scalar field $\Upsilon(X, x, y)$ obeys the following equation of motion:

$$
\left[-\square_{(D+1)}+m^{2}+\xi \mathcal{R}_{(D+1)}\right] \Upsilon=0
$$

where $\mathcal{R}_{(D+1)}$ is the higher dimensional curvature and $\square_{(D+1)}$ is the D'Alembertian, both computed from the metric $(1)$.

Using the explicit expression for the metric tensor, we can disentangle, in equation (16), the dependence on the internal manifold from the Minkowskian one. A straightforward calculation gives:

$$
\begin{aligned}
& {\left[-e^{-2 \sigma} \square-e^{-2 \rho} \frac{1}{R^{2}} \Delta_{(\gamma)}-e^{-\tau} \partial_{y} e^{\tau} \partial_{y}+\right.} \\
& \left.\quad+m^{2}+\xi e^{-2 \rho} \frac{1}{R^{2}} \mathcal{R}^{(\gamma)}-\xi F(y)\right] \Upsilon=0,
\end{aligned}
$$

where $\Delta_{(\gamma)}$ is the laplacian related to $\gamma_{i j}, \square$ is the $D_{1}$ dimensional flat D'Alembertian, and

$$
\begin{aligned}
F(y) & =2 \tau^{\prime \prime}(y)+\tau^{\prime}(y)^{2}+D_{1} \sigma^{\prime}(y)^{2}+D_{2} \rho^{\prime}(y)^{2}, \\
\tau(y) & =D_{1} \sigma(y)+D_{2} \rho(y) .
\end{aligned}
$$

We now expand the field $\Upsilon(x, X, y)$ in terms of a complete set of modes carrying a momentum along the orbifold and $\Sigma$ directions labelled by indexes $n$ and $l$ respectively,

$$
\Upsilon(x, X, y)=\sum_{l, n} \Psi_{l}(X) \Phi_{l, n}(x) Z_{l, n}(y) .
$$

Here, the modes $\Psi_{l}(X)$ are a complete set of solutions of the Klein-Gordon equation on the manifold $\Sigma$ :

$$
P_{\Sigma} \Psi_{l}(X) \equiv \frac{1}{R^{2}}\left[-\Delta_{(\gamma)}+\xi \mathcal{R}_{(\gamma)}\right] \Psi_{l}(X)=\lambda_{l}^{2} \Psi_{l}(X),
$$

with eigenvalues $\lambda_{l}^{2}$ and degeneracy ${ }^{3} g_{l}$. If we now require $\Phi_{l, n}(x)$ to satisfy the Klein-Gordon equation on the Minkowskian factor of the space-time $\mathcal{M}$ with masses $m_{l, n}^{2}$,

$$
\left[-\square+m_{l, n}^{2}\right] \Phi_{l, n}(x)=0
$$

\footnotetext{
${ }^{3}$ Although we assume $P_{\Sigma}$ to be either positive semidefinite or positive definite, the label $l=0$ always refers to the zero eigenvalue, i.e., $\lambda_{0}=0$, the existence of this eigenvalue being set by $g_{0}$ being 0 or 1 .
} 
we are left with a radial equation for the modes $Z_{l, n}(y)$ of the form

$$
e^{2 \sigma}\left[-e^{-\tau} \partial_{y} e^{\tau} \partial_{y}+m^{2}-\xi F(y)+\lambda_{l}^{2} e^{-2 \rho}\right] Z_{l, n}=m_{l, n}^{2} Z_{l, n}
$$

This equation is valid for any warp factors $\sigma$ and $\rho$, and can be viewed as an eigenvalue problem for the orbifold modes $Z_{l, n}$ and the physical masses $m_{l, n}$. Both of them depend in general on the 'internal' index $l$. In this paper we consider the case of two equal warp factors, with

$$
\rho(y)=\sigma(y)=-k|y|
$$

Defining $D=D_{1}+D_{2}$, we can specialize Eq. (22) to this case as

$$
\left[-e^{(2-D) \sigma} \partial_{y} e^{D \sigma} \partial_{y}+m^{2} e^{2 \sigma}-\xi F(y) e^{2 \sigma}\right] Z_{l, n}=\left(m_{l, n}^{2}-\lambda_{l}^{2}\right) Z_{l, n} .
$$

We note that the operator in the l.h.s. does not depend on the internal index $l$. Accordingly, in this case neither the modes $Z_{l, n}$ nor the combination $q_{n}^{2} \equiv m_{l, n}^{2}-\lambda_{l}^{2}$ depend on $l$. In other words, the dependence on $l$ and $n$ of the masses is factorized for this geometry,

$$
m_{l, n}^{2}=q_{n}^{2}+\lambda_{l}^{2}
$$

Therefore, from now on we shall drop this index in $Z$. On the other hand, Eq. (23) is similar to the one which arises in the RS model, and the most general solution can still be written in terms of Bessel functions:

$$
Z_{n}^{\beta}(y)=\epsilon_{\beta}(y)\left[A_{n}^{\beta} J_{\nu}\left(\frac{q_{n}}{k} e^{-\sigma}\right)+B_{n}^{\beta} Y_{\nu}\left(\frac{q_{n}}{k} e^{-\sigma}\right)\right]
$$

where for notational convenience we have defined

$$
\epsilon_{\beta}(y)=e^{-\frac{D}{2} \sigma(y)}\left\{\begin{array}{lc}
y /|y| & \beta=\text { twisted } \\
1 & \beta=\text { untwisted }
\end{array}\right.
$$

and

$$
\nu^{2}=\frac{m^{2}}{k^{2}}-D(D+1) \xi+\frac{D^{2}}{4} .
$$

The index $\beta$ has been introduced in order to discriminate the two possible cases of $\Upsilon$ being untwisted $\left(Z_{n}(-y)=Z_{n}(y)\right)$ or twisted $\left(Z_{n}(-y)=-Z_{n}(y)\right)$. Imposing the appropriate boundary conditions, which can be obtained by integrating equation (23) across the orbifold fixed points, we find that the eigenvalues $q_{n}$ are determined by the transcendental equation:

$$
F_{\nu}^{\beta}\left(\frac{q_{n}}{k a}\right)=0
$$

where

$$
F_{\nu}^{\beta}(z)=\left\{\begin{array}{l}
Y_{\nu}(a z) J_{\nu}(z)-J_{\nu}(a z) Y_{\nu}(z) \quad \beta=\text { twisted } \\
y_{\nu}(a z) j_{\nu}(z)-j_{\nu}(a z) y_{\nu}(z) \quad \beta=\text { untwisted }
\end{array}\right.
$$

As in the RS model, the combinations of Bessel functions relevant to the untwisted case are given by

$$
\begin{aligned}
& j_{\nu}(z)=\frac{1}{2} D(1-4 \xi) J_{\nu}(z)+z J_{\nu}^{\prime}(z), \\
& y_{\nu}(z)=\frac{1}{2} D(1-4 \xi) Y_{\nu}(z)+z Y_{\nu}^{\prime}(z),
\end{aligned}
$$

This completes the Kaluza-Klein reduction of the bulk scalar field.

In the following we will report only on the case of untwisted fields, although the case of twisted fields can be obtained at ease with simple modifications of our calculation. 


\section{QUANTUM EFFECTIVE ACTION}

The one loop effective action $\Gamma$ can be expressed as the sum over the contributions of each mode, $\Gamma_{l, n}$

$$
\Gamma=\sum \Gamma_{l, n} .
$$

The previous expression can be evaluated in a variety of ways (see for instance [9,33]). Dimensional regularization of the 4 -dimensional Minkowski directions to $4-2 \epsilon$ leads to the following expression for the vacuum energy contribution to the effective action

$$
\Gamma=-\int d^{4-2 \epsilon} x V^{\mathrm{reg}}(s)
$$

with

$$
V^{\mathrm{reg}}(s)=-\frac{1}{2}(4 \pi)^{s} \mu^{2 \epsilon} \Gamma(s) \sum_{n, l}^{\prime} g_{l}\left(q_{n}^{2}+\lambda_{l}^{2}\right)^{-s}
$$

where the prime in the sum assumes that the zero mass mode is excluded (since it does not contribute) and $s=-2+\epsilon$.

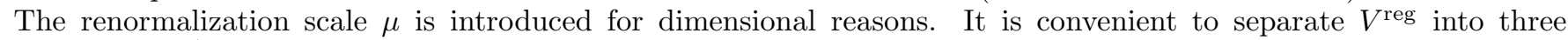
contributions $^{4}$

$$
V^{\mathrm{reg}}(s)=V_{\Sigma}(s)+V_{R S}(s)+V_{*}(s)
$$

where

$$
\begin{aligned}
V_{\Sigma}(s) & =-\frac{\mu^{2 \epsilon}}{2(4 \pi)^{-s}} \Gamma(s) \sum_{l=1}^{\infty} g_{l} \lambda_{l}^{-2 s}, \\
V_{R S}(s) & =-g_{0} \frac{\mu^{4}}{2(4 \pi)^{-s}}(k a / \mu)^{-2 s} \Gamma(s) \sum_{n=1}^{\infty} x_{n}^{-2 s}, \\
V_{*}(s) & =-\frac{\mu^{4}}{2(4 \pi)^{-s}}(k a / \mu)^{-2 s} \Gamma(s) \sum_{n, l=1}^{\infty} g_{l}\left(x_{n}^{2}+y_{l}^{2}\right)^{-s},
\end{aligned}
$$

with $x_{n}=q_{n} / k a$ and $y_{l}=\lambda_{l} / k a$. Thus, $V_{\Sigma}$ retains the contributions from the orbifold zero mode (present only for an untwisted field), $V_{R S}$ is the contribution from the $\Sigma$ zero mode (which coincides with the potential in the RS model), and $V_{*}$ includes the contribution from mixed states. It is clear from Eqs. (28) and (29) that $x_{n}$ depends on $a$ only. Since $\lambda_{l}$ scales like $1 / R$, we can factor out the dependence on this modulus, defining dimensionless eigenvalues $\hat{\lambda}_{l}=R \lambda_{l}$, that do not depend on $R$. If $\Sigma$ is a one-parameter space, then $\lambda_{l}$ cannot depend on any other shape moduli. However, here we are interested in the dependence on the breathing mode $R$ only. So, in general, $y_{l}=\hat{\lambda}_{l} /(k a R)$ depends on the moduli described in Sec. (II B) through $R_{-}$.

The first term in (32) $V_{\Sigma}$ results from the KK excitations along the internal manifold. It can be expressed in terms of the generalized $\zeta$ function associated to the laplacian $P_{\Sigma}$ defined on $\Sigma$ (see Eq. (20)), ${ }^{5}$

$$
\zeta(s) \equiv \zeta\left(s \mid P_{\Sigma}\right)=\sum_{l=1}^{\infty} g_{l} \hat{\lambda}_{l}^{-2 s} .
$$

using the previous rescaling we can recast $V_{\Sigma}$ as

$$
V_{\Sigma}(-2+\epsilon)=-\frac{1}{32 \pi^{2} R^{4}}(\mu R)^{2 \epsilon} \Gamma(-2+\epsilon) \zeta(-2+\epsilon)
$$

\footnotetext{
${ }^{4}$ Here we define $\lambda_{0}=0$, so that the existence of such a zero eigenvalue or not is controlled by $g_{0}$. If $g_{0} \neq 0$, the RS contribution comes about explicitly and introduces a divergence which needs to be cancelled by a corresponding contribution coming from $V_{*}$. This cancellation provides a non-trivial check of our evaluation. The case of a strictly positive definite operator, can be obtained by putting $g_{0}$ to zero.

${ }^{5}$ The fact that this term does not depend on the eigenvalues $q_{n}$ is a consequence of how we have performed the Kaluza-Klein reduction.
} 
where we redefined the renormalization constant $\mu$. The previous expression can be elegantly dealt with by using the Mittag-Leffler representation for the $\zeta$ function, which proves to be a very useful tool to handle the pole structure of the $\zeta$ function, since the residues at the poles are determined by geometrical quantities of $\Sigma$ (See for example [34]). As shown in appendix A,

$$
\zeta(s)=\frac{1}{\Gamma(s)}\left\{\sum_{p=0}^{\infty} \frac{\tilde{C}_{p}}{s-D_{2} / 2+p}+f(s)\right\},
$$

where $\tilde{C}_{p}=C_{p}-g_{0} \delta_{p, D_{2} / 2}$ and $C_{p}$ are the (integrated) Seeley-DeWitt coefficients of the operator $P_{\Sigma}$ on $\Sigma, p$ runs over the positive half integers and $f(s)$ is an entire function. In fact, the sum (38) runs over half integers, but, since $\Sigma$ has no internal boundaries, the coefficients $C_{i / 2}$ are zero. Relation (38) can now be used to regulate $V_{\Sigma}$, and a simple calculation gives

$$
V_{\Sigma}(s=-2+\epsilon)=-\frac{1}{32 \pi^{2} R^{4}}\left(4 \pi R^{2} \mu^{2}\right)^{\epsilon}\left[\Omega_{-2}+C_{D_{2} / 2+2} \frac{1}{\epsilon}\right]
$$

where $\Omega_{-2}$ is the constant term in the power series of $\Gamma(s) \zeta(s)$ around $s=-2$ (see Eq. (A17)).

The term proportional to the RS contribution has been computed in $[10,13,16]$. Without going into details, we write such term as follows:

$$
V_{R S}=-g_{0} \frac{k^{4}}{32 \pi^{2}}(k / \mu)^{-2 \epsilon}\left\{-d_{4} \frac{1}{\epsilon}\left(1+a^{4-2 \epsilon}\right)+c_{1}+a^{4} c_{2}-2 a^{4} \mathcal{V}(a)\right\},
$$

where we have introduced

$$
\mathcal{V}(a)=\int_{0}^{\infty} d z z^{3} \ln \left(1-\frac{k_{\nu}(z)}{k_{\nu}(a z)} \frac{i_{\nu}(a z)}{i_{\nu}(z)}\right)
$$

and the coefficients $c_{1}$ and $c_{2}$ do not depend on $a$. Here, the coefficient $d_{4}$ depends on the mass and non-mininal coupling of $\Upsilon$, and is defined through Eqs. $(51,47,45)$.

Before entering into the discussion of the higher dimensional contribution due to the mixed KK states $V_{*}$, we can foresee now some of the details of the computation. As mentioned above, the case when $\Sigma$ is a torus corresponds to a toroidal compactification of a slice of higher dimensional $A d S$ space. Since it is a maximally symmetric space, all the geometric invariants are constant, and so proportional to the brane tensions. Thus, the only possible divergence that can appear is of the form $\int d^{4} x\left(1+a^{D}\right)$. However, regardless of the dimension of $\Sigma$, the contribution from $V_{R S}$ contains a divergence of the form $\int d^{4} x\left(1+a^{4}\right)$. Of course, what happens is that aside from the higher dimensional divergence, $V_{*}$ also contains another divergence that cancels the RS one. This feature occurs not only when $\Sigma$ is a torus. Rather, it is completely general. As we show next and in appendix A, the divergence of $V_{*}+V_{R S}$ proportional to $\int d^{4} x\left(1+a^{4}\right)$ is always controlled by a geometric invariant related to $\Sigma$ (which trivially vanishes for a torus). This ensures that if this divergence persists, it is because one can build some operator that behaves like it in this background.

Let us now turn to the evaluation of $V_{*}$. First of all, let us concentrate on the sum

$$
\Gamma(s) \sum_{n, l=1}^{\infty} g_{l}\left(x_{n}^{2}+y_{l}^{2}\right)^{-s} .
$$

This is not straightforward to compute, however the method developed in [35-37] allows us to perform such a calculation. Since, in our case, the evaluation does not present any particular difficulty, we will be brief and address the reader to the original references for an introduction to the details of the method.

The residue theorem permits us to express the sum (42) as a contour integral and an appropriate choice of the contour of integration leaves us with

$$
\Gamma(s) \frac{\sin (\pi s)}{\pi} \sum_{l} g_{l} \int_{y_{l}}^{\infty}\left(x^{2}-y_{l}^{2}\right)^{-s} \frac{d}{d x} \ln \left[F_{\nu}(i x)\right] d x
$$

which, by changing variable and by using some known properties of the Bessel functions can be recast as

$$
\frac{1}{\Gamma(1-s)} \sum_{l} g_{l} y_{l}^{-2 s} \int_{1}^{\infty}\left(z^{2}-1\right)^{-s} \frac{d}{d z} \ln \left[P_{\nu}\left(y_{l} z\right)\right] d z,
$$


where

$$
P_{\nu}(z)=F_{\nu}(i z)=\frac{2}{\pi}\left[k_{\nu}(z) i_{\nu}(a z)-k_{\nu}(a z) i_{\nu}(z)\right]
$$

and

$$
\begin{aligned}
& i_{\nu}(z)=z I_{\nu}^{\prime}(z)+\frac{1}{2} D(1-4 \xi) I_{\nu}(z) \\
& k_{\nu}(z)=z K_{\nu}^{\prime}(z)+\frac{1}{2} D(1-4 \xi) K_{\nu}(z) .
\end{aligned}
$$

The integral (43) is considered in [38] for $s=0$. In that case, the result can be expressed in closed form. However, for $s=-2$ this does not seem to be possible.

We can regulate relation (44) using the asymptotic expansions for the Bessel functions. The large $z$ behaviour of $i_{\nu}$ and $k_{\nu}$ can be written as (see e.g. [10])

$$
\begin{aligned}
i_{\nu}(z) & =\sqrt{\frac{z}{2 \pi}} e^{z} \Theta^{(i)}(z), \\
k_{\nu}(z) & =\sqrt{\frac{\pi z}{2}} e^{-z} \Theta^{(k)}(z),
\end{aligned}
$$

where $\Theta^{(k)}(z)=\Theta^{(i)}(-z)$ is a power series in $1 / z$ beginning with 1. Thus, we can recast the integrand of Eq. (44) in the form

$$
P_{\nu}\left(y_{l} z\right)=-\frac{\sqrt{a}}{\pi} y_{l} z e^{(1-a) y_{l} z} \Theta^{(i)}\left(y_{l} z\right) \Theta^{(k)}\left(a y_{l} z\right)\left[1-\frac{k_{\nu}\left(y_{l} z\right) i_{\nu}\left(a y_{l} z\right)}{i_{\nu}\left(y_{l} z\right) k_{\nu}\left(a y_{l} z\right)}\right] .
$$

Up to a constant term, $\ln P_{\nu}$ can be split as

$$
\ln \left[P_{\nu}\left(y_{l} z\right)\right]=\mathcal{H}_{l}^{(1)}(z)+\mathcal{H}_{l}^{(2)}(z)+\mathcal{H}_{l}^{(3)}(z)
$$

with

$$
\begin{aligned}
& \mathcal{H}_{l}^{(1)}(z)=\ln z+(1-a) y_{l} z \\
& \mathcal{H}_{l}^{(2)}(z)=\ln \left[\Theta^{(i)}\left(y_{l} z\right) \Theta^{(k)}\left(a y_{l} z\right)\right], \\
& \mathcal{H}_{l}^{(3)}(z)=\ln \left[1-\frac{k_{\nu}\left(y_{l} z\right) i_{\nu}\left(a y_{l} z\right)}{i_{\nu}\left(y_{l} z\right) k_{\nu}\left(a y_{l} z\right)}\right] .
\end{aligned}
$$

and correspondingly,

$$
V_{*}(s)=V_{*}^{(1)}(s)+V_{*}^{(2)}(s)+V_{*}^{(3)}(s),
$$

with

$$
V_{*}^{(\alpha)}=-\frac{\mu^{4}}{2(4 \pi)^{-s}}(k a / \mu)^{-2 s} \frac{1}{\Gamma(1-s)} \sum_{l=1}^{\infty} g_{l} y_{l}^{-2 s} \int_{1}^{\infty}\left(z^{2}-1\right)^{-s} \frac{d}{d z} \ln \left[\mathcal{H}_{l}^{(\alpha)}(z)\right] d z \quad(\alpha=1,2,3) .
$$

The evaluation of $V_{*}^{(1)}(s)$ is analogous to the one for $V_{\Sigma}(s)$ and, once more, the Mittag-Leffler expansion allows to express the result in terms of the heat-kernel coefficients of the operator $P_{\Sigma}$ on $\Sigma$. We find

$$
\begin{aligned}
V_{*}^{(1)}(s=-2+\epsilon)=-\frac{1}{32 \pi^{2} R^{4}}(\mu R)^{2 \epsilon} & \left\{\left[\frac{1}{2} C_{2+D_{2} / 2}+\frac{1}{2 \sqrt{\pi}} C_{5 / 2+D_{2} / 2} \frac{1-a}{k a R}\right] \frac{1}{\epsilon}\right. \\
& \left.+\frac{1}{2} \Omega_{-2}+\frac{1}{2 \sqrt{\pi}} \Omega_{-5 / 2} \frac{1-a}{k a R}\right\}
\end{aligned}
$$


The second term $V_{*}^{(2)}(s)$ can be evaluated ${ }^{6}$ using the explicit form of $\Theta^{(i)}$ and $\Theta^{(k)}$ :

$$
\ln \left(\Theta^{(i)}(z) \Theta^{(k)}(a z)\right) \simeq \sum_{j=1}^{\infty}\left(1+\frac{(-1)^{j}}{a^{j}}\right) d_{j} z^{-j} \quad \text { for } \quad z \gg 1,
$$

the coefficients $d_{j}$ can be obtained by simply Taylor expanding the logarithm. Using (51) and treating the sum over the eigenvalues $y_{l}$ as in the case of $V_{\Sigma}$ (see App. (A)), we can write $V_{*}^{(2)}$ as

$$
V_{*}^{(2)}(s=-2+\epsilon)=\frac{1}{32 \pi^{2} R^{4}}(\mu R)^{2 \epsilon} \sum_{j=1}^{\infty} \frac{d_{j}}{\Gamma(j / 2)}\left\{\left[C_{2+D_{2} / 2-j / 2}-g_{0} \delta_{4, j}\right] \frac{1}{\epsilon}+\Omega_{-2+j / 2}\right\}\left((k a R)^{j}+(-k R)^{j}\right) .
$$

The third term in $V_{*}^{(3)}(s)$ is finite by construction, and we can put safely $s=-2$,

$$
V_{*}^{(3)}(s=-2)=-\frac{1}{64 \pi^{2} R^{4}} \sum_{l=1}^{\infty} g_{l} \hat{\lambda}_{l}^{4} \int_{1}^{\infty}\left(z^{2}-1\right)^{2} \frac{d}{d z} \mathcal{H}_{l}^{(3)}(z) d z
$$

Combining the previous results, we obtain the unrenormalized Casimir energy:

$$
\begin{aligned}
V^{\mathrm{reg}}=-\frac{1}{32 \pi^{2} R^{4}} & {\left[\sum_{j=-1}^{\infty}\left[\left(k R_{-}\right)^{j}+\left(-k R_{+}\right)^{j}\right]\left\{\gamma_{j}+\left(\beta_{j}-g_{0} d_{4} \delta_{4, j}\right) \frac{1}{\epsilon}(\mu R)^{2 \epsilon}\right\}\right.} \\
& \left.+g_{0}(k R)^{4}\left\{c_{1}+a^{4} c_{2}-2 a^{4} \mathcal{V}(a)-\frac{1}{\epsilon}\left(1+a^{4-2 \epsilon}\right)(k / \mu)^{-2 \epsilon}\right\}+2 \sum_{l=1}^{\infty} g_{l} \hat{\lambda}_{l}^{4} \mathcal{V}_{l}\left(a, R_{-}\right)\right]
\end{aligned}
$$

where

$$
\beta_{j}=\left\{\begin{array}{lll}
(1 / 2 \sqrt{\pi}) C_{5 / 2+D_{2} / 2} & \text { for } & j=-1 \\
(3 / 2) C_{2+D_{2} / 2} & \text { for } & j=0 \\
-\left(d_{j} / \Gamma(j / 2)\right) C_{2-j / 2+D_{2} / 2} & \text { otherwise }
\end{array}\right.
$$

and we understand that the Seeley-DeWitt coefficients $C_{i}$ are zero if $i<0$,

$$
\gamma_{j}=\left\{\begin{array}{lll}
(1 / 2 \sqrt{\pi}) \Omega_{-5 / 2} & \text { for } & j=-1 \\
(3 / 2) \Omega_{-2} & \text { for } & j=0 \\
-\left(d_{j} / \Gamma(j / 2)\right) \Omega_{j / 2-2} & \text { otherwise }
\end{array}\right.
$$

and

$$
\mathcal{V}_{l}\left(a, R_{-}\right)=\int_{1}^{\infty} d z z\left(z^{2}-1\right) \ln \left(1-\frac{k_{\nu}\left(y_{l} z\right)}{k_{\nu}\left(y_{l} a z\right)} \frac{i_{\nu}\left(y_{l} a z\right)}{i_{\nu}\left(y_{l} z\right)}\right) .
$$

Equation (54) shows that as we advanced above, the lower dimensional divergence coming from the RS contribution $V_{R S}$ is always cancelled, independently of the structure of the internal manifold $\Sigma$. On the other hand, the contribution from the KK modes along $\Sigma$ only (lower dimensional, as well) may give a divergence corresponding to $j=0$. This is controlled by the Seeley-DeWitt coefficient $C_{D_{2} / 2+2}$, and gives $1 / 2$ of the resulting $3 / 2$ factor in $\beta_{0}$, the rest coming from the mixed states in $V_{*}$. In particular, if $D_{2}$ is odd, then there is no such divergence (if $\Sigma$ is boundaryless), in accordance with the absence of any operator that behaves as $\int d^{4} x 1 / R^{4}$ in the background, in this case.

To conclude this section, we briefly comment on the differences appearing when we consider a twisted bulk field. First, since there is no orbifold zero mode, its contribution $V_{\Sigma}$ is not present. One can show that the asymptotic behaviour of the function $P_{\nu}$ differs in two powers of the argument, originating a change of sign in the contribution to $\beta_{0}$ and $\gamma_{0}$ from $V_{*}^{(1)}$. Of course, the $d_{j}$ coefficients also change, and can be read from $[10,13]$. In brief, one needs to change the $d_{j}$ by the corresponding one, and the $3 / 2$ factor in $\beta_{0}$ and $\gamma_{0}$ by $-1 / 2$. Also, we haven't included any brane mass terms or kinetic terms, relevant for the untwisted case only (aside from the ones arising from the coupling to curvature). In principle, these can be different on each brane. This changes our result in that we would have different coefficients, $d_{j}^{ \pm}$, for the $r_{ \pm}$series.

\footnotetext{
${ }^{6}$ Strictly speaking we are using an asymptotic expansion and therefore the equality sign is not exact. However, the approximation we are making is reasonable because the integration range vary from 1 to $\infty$ and the argument of $\Theta^{(i)}$ and $\Theta^{(k)}$ is large in the region $R \ll 1$ and $a R \ll 1$.
} 


\section{RENORMALIZATION}

In the previous section, we have computed the unrenormalized Casimir energy (54) using dimensional regularization. This allows us to isolate the divergent terms, of the form

$$
\Gamma^{\mathrm{div}}=\frac{1}{\epsilon} \frac{1}{32 \pi^{2}} \frac{1}{R^{4}} \int d^{4} x \sum_{j=-1}^{D_{2}+4} \beta_{j}\left(a^{j}+(-1)^{j}\right)(k R)^{j},
$$

with $\beta_{j}$ given by (55). A finite number of divergences appear because we have computed the one loop contribution to the effective potential.

It is well known (see e.g. [37]) that the divergences present in the effective action are given by the Seeley-DeWitt coefficient $C_{(D+1) / 2}$ related to the operator in (16) on our $D+1$ dimensional background space-time. Since this has boundaries, nonzero boundary terms are present for any dimension. Moreover, since the extrinsic curvature is constant in the space-time we are considering, several powers of the intrinsic curvature of the boundaries are present. Finally, it is easily shown that once any possible bulk term is evaluated on the background solution, it can be recast as boundary term for this specific solution. ${ }^{7}$

So, we shall consider boundary term of the form

$$
\sum_{ \pm} \int d^{D} x \sqrt{g_{(D) \pm}} \mathcal{R}_{(D) \pm}^{N}
$$

where $N=0,1,2, \ldots$ and $\mathcal{R}_{(D) \pm}$ denotes the (intrinsic) curvature computed from the induced metrics on the branes $g_{(D) \pm}$. Using the explicit expression for the metric tensor (1), a simple calculation shows the previous term generates a contribution proportional to $R^{D_{2}-2 N}$ coming from the brane at $y_{+}$, and a contribution of the form $R^{D_{2}-2 N} a^{4+D_{2}-2 \epsilon-2 N}$ from the other brane. Then, it is clear that all the divergences in (58) can be dealt with operators of the form (59). Specifically, we can take the following expression as the counter-term needed to renormalize the effective action:

$$
\begin{aligned}
S_{j}^{C T} & =\frac{1}{32 \pi^{2} \epsilon} \int d^{D_{2}} X d^{4-2 \epsilon} x\left\{\sqrt{g_{(D)+}} \kappa_{j}^{+} \mathcal{R}_{+}^{\left(D_{2}+4-j\right) / 2}+\sqrt{g_{(D)-}} \kappa_{j}^{-} \mathcal{R}_{-}^{\left(D_{2}+4-j\right) / 2}\right\} \\
& =\frac{1}{32 \pi^{2} \epsilon} \int d^{4-2 \epsilon} x \frac{R^{j}}{R^{4}}\left\{\kappa_{j}^{+}+\kappa_{j}^{-} a^{j-2 \epsilon}\right\}
\end{aligned}
$$

The index $j$ here runs over the integers comprised between -1 and $4+D_{2}$, and $\kappa_{j}^{ \pm}$are renormalization constants. We recall that, from (58) and (55), the divergences occur for $j$ even only if $D_{2}$ is even, and for $j$ odd when $D_{2}$ odd.

In the process of subtracting the counter-terms, finite contributions to the vacuum energy with a logarithmic dependence on the moduli are generated. The renormalized expression can be written as

$$
\begin{aligned}
V\left(R_{ \pm}\right)=-\frac{1}{32 \pi^{2} R^{4}} & {\left[\sum _ { j = - 1 } ^ { \infty } \left\{\left(\beta_{j}-g_{0} d_{4} \delta_{4, j}\right)\left[\left(k R_{-}\right)^{j} \ln \left(k R_{-}\right)^{2}+\left(-k R_{+}\right)^{j} \ln \left(k R_{+}\right)^{2}\right]\right.\right.} \\
& \left.+\left(\gamma_{j}-\beta_{j} \ln (k / \mu)^{2}\right)\left[\left(k R_{-}\right)^{j}+\left(-k R_{+}\right)^{j}\right]\right\} \\
& \left.+g_{0}(k R)^{4}\left\{c_{1}+a^{4} c_{2}-2 a^{4} \mathcal{V}(a)\right\}+2 \sum_{l=1}^{\infty} g_{l} \hat{\lambda}_{l}^{4} \mathcal{V}_{l}\left(a, R_{-}\right)\right]
\end{aligned}
$$

A few remarks are now in order. First of all, note that we recast the result in order to isolate the $\mu$ dependent terms. Such terms are not computable from our effective theory, rather they have to be fixed by imposing a set of renormalization conditions. Secondly, notice that the result is valid for $D_{2}$ even as well as for $D_{2}$ odd, and the heat-kernel coefficients automatically take this into account. Also, it should be noted that the flat space limit $k \rightarrow 0$ is well defined. One has to take into account that $R_{+}=R e^{-k y_{+}}$and a similar expression for $R_{-}$. Then in the limit

\footnotetext{
${ }^{7}$ For instance, $\int d^{(D+1)} x \sqrt{g_{(D+1)}} \Lambda=\sum_{ \pm} \int d^{D} x \sqrt{g_{(D) \pm}} \sigma_{ \pm}$, with $\sigma_{ \pm}=\mp 2 \Lambda / D k$.
} 
$k \rightarrow 0$, keeping the proper distance between two branes $\left(y_{-}-y_{+}\right)$fixed, all terms which would be singular in $k$ cancel each other in the first and second lines of Eq. (61). The rest of the terms are well behaved. The resulting expression is proportional to $\left(y_{-}-y_{+}\right) / R^{5}$, which has a clear interpretation in terms of the Casimir energy.

An important remark concerns the divergence proportional to $R_{+}^{4}+R_{-}^{4}$. This is the divergence present in the RS contribution $[10,13]$. For $D_{2}$ odd, it is not reproduced by any of the counter-terms in $(59)$. However, this is not a problem because such divergence is cancelled by the corresponding one coming from (52) for $j=4$.

\section{PHYSICAL SCALES AND THE EW/PLANCK HIERARCHY}

In this section we propose a scenario where supersymmetry is broken at a scale $\eta_{\mathrm{SUSY}}$ not far below the cutoff scale $M$, and the hierarchy between the electroweak and the effective Planck scales is generated by a combination of redshift and large volume effects. Also, we discuss the range of possible values for the dynamical (the moduli $R_{ \pm}$) and the fixed scales (the cutoff $M$ and the SUSY breaking scale $\eta_{\mathrm{SUSY}}$ ).

From Eqs. (12) and (11), we see that the relation between the four dimensional effective Planck mass and the higher dimensional one (in the four dimensional effective theory using the Einstein frame metric $\hat{g}_{\mu \nu}$ ) is

$$
m_{P}^{2} \approx(M R)^{D_{2}} \frac{M}{k} M^{2} .
$$

We shall assume that the masses of particles (located at $y=y_{-}$) are somewhat below the cutoff $M$. In the four dimensional theory, these masses are redshifted down to $\sim a M$. Then, the EW/Planck hierarchy is given by

$$
h^{2} \equiv a^{2} \frac{M^{2}}{m_{P}^{2}} \sim \frac{a^{2}}{(R M)^{D_{2}}} \frac{k}{M} \sim 10^{-32} .
$$

Thus, the EW/Planck hierarchy $h$ is explained in this model due to a combination of redshift [6] and large volume [3] effects (even though the branes are of codimension 1). The crucial ingredient in order for the large volume effect to be efficient (aside from having a long orbifold), is that the additional extra space $\Sigma$ exponentially grows as one moves away from the negative tension brane (see Fig. 1). In this way, matter is allowed to propagate along a small $\Sigma$, of size $R_{-}$, whereas gravity is diluted since it propagates through a much larger $\Sigma$, of effective size $R_{+}$. Since the gauge interactions must not be diluted by an analogous effect, we have to assume that the compactification scale on the negative tension brane $1 / R_{-}$is close to the fundamental cutoff $M .{ }^{8}$

Our model solves the hierarchy problem in a fashion very similar to the models considered in $[39,40]$, with two concentric branes embedded in a noncompact bulk. In this references, the hierarchy and the positions of the branes are naturally stabilized by a generalization of the Goldberger and Wise mechanism [8] (see also [41]).

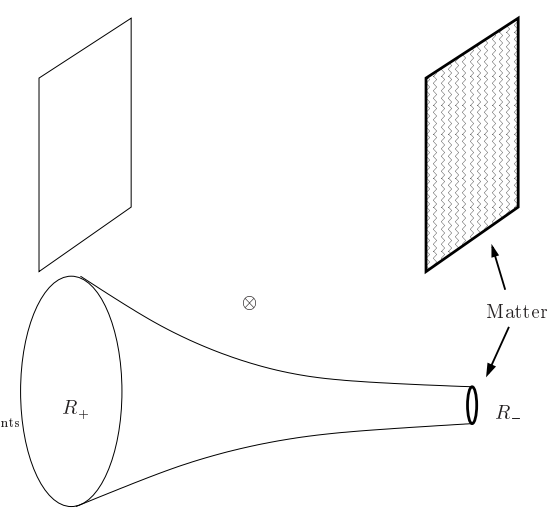

FIG. 1. Matter can propagate along the additional extra space $\Sigma$ of size $R_{-}$, but gravity samples a much bigger space.

\footnotetext{
${ }^{8}$ Keeping only the $\Sigma$-zero mode in the action for a $D$ dimensional Yang-Mills field $F_{A B}$ at $y=y_{-}$with coupling constant $g_{*(D)}^{2} \sim M^{4-D}$, one obtains $\int d^{D} x \sqrt{g_{(D)-}} \frac{1}{g_{*(D)}^{2}} F_{A B} F_{C D} g_{(D)-}^{A C} g_{(D)-}^{B D} \simeq \int d^{4} x \sqrt{\hat{g}} R_{-}^{D_{2}} \frac{1}{g_{*(D)}^{2}} F_{\mu \nu} F_{\rho \sigma} \hat{g}^{\mu \rho} \hat{g}^{\nu \sigma}$, where we used that $\tilde{g}_{\mu \nu} \simeq \hat{g}_{\mu \nu}$. Thus, the four dimensional YM coupling is identified as $g_{*(4)}^{2}=g_{*(D)}^{2} / R_{-}^{D_{2}} \sim 1 /\left(M R_{-}\right)^{D_{2}}$.
} 
Let us now examine the constraints that we have on the moduli and the physical values they can take. First of all, we are thinking of an inter-brane distance $d=\left|y_{-}-y_{+}\right|$somewhat larger than the inverse curvature scale $1 / k$ of the bulk, in order to have a substantial redshift factor $a=e^{-k d}$. On the other hand, the smallest physical length scale is given by the size of $\Sigma$ at the negative tension brane, $R_{-}$. This cannot be smaller than the fundamental length of the theory $M^{-1}$ though, as argued in the previous paragraph, it should be close to it. There is a tighter technical restriction which we shall use so that the result for the potential (61) is valid. This, is organized as a power series in $r_{ \pm}=k R_{ \pm}$, and can be trusted only when $1 / R_{+}$a bit larger than the curvature scale and $1 / R_{-}$a factor $a$ above (recall that the ratio $R_{-} / R_{+}$coincides with the redshift factor $a$ ). Incidentally, we remark that this corresponds to the physical situation where the size of the internal manifold $\Sigma$ is everywhere smaller than the inter-brane distance $\sim 1 / k .{ }^{9}$ So, we must assume a separation between the fundamental cutoff $M$ and the curvature scale $k$ at least of order $a$. This leads to the following scenario.

Consider a supersymmetric theory where the SUSY breaking scale is given by $\eta_{\mathrm{SUSY}}$. Then, the bulk cosmological constant $\Lambda \sim k^{2} M^{D-1}$ is expected to be proportional to $\eta_{\mathrm{SUSY}}^{D+1}$, which leads to

$$
k \sim\left(\frac{\eta_{\mathrm{SUSY}}}{M}\right)^{(D+1) / 2} M \ll M
$$

Even if SUSY is broken not far below the cut-off scale, this may lead to a curvature scale $k$ many orders of magnitude below $M$, due to the large exponent in (64). If the moduli $R_{ \pm}$are stabilized near the values $R_{+} \sim 1 / k$ and $R_{-} \sim 1 / M$, then $a \sim k / M$ and from (63), the hierarchy is given by

$$
h \sim\left(\frac{k}{M}\right)^{(D-1) / 2} \sim\left(\frac{\eta_{\mathrm{SUSY}}}{M}\right)^{\left(D^{2}-1\right) / 4} .
$$

Note that the required hierarchy is obtained with $\eta_{\mathrm{SUSY}}$ within one order of magnitude of the cut-off $M$ for $D=10$, and less than 3 orders of magnitude below $M$ for $D=5$.

This shows how the problem of the stabilization of a large hierarchy works in this model. Having introduced a small separation between the SUSY breaking and the cutoff scales, we obtain a stable very flat warped space-time, $k \ll M$. If the potential (61) can stabilize the moduli $R_{ \pm}$near the values, $R_{+} \sim 1 / k$ and $R_{-} \sim 1 / M$, then the effective Planck mass is very large as compared to the EW scale. Whether or not the effective potential (61) can do this job is addressed in next section.

Let us discuss the physical scales in the model in some detail. As illustrated in Fig. 1, the branes are of codimension 1 , so that matter (residing on the negative tension brane, at $y=y_{-}$) can propagate through a physical extra dimensional space of size $\sim R_{-}$. The mass scales on this brane are redshifted by a factor $a$, thus the mass of the first KK excitations of matter fields is $1 / R$. Then, from collider physics, we have to set the compactification scale $1 / R \gtrsim T e V$, at least.

In contrast, gravity propagates through the whole bulk space, and its KK spectrum is analogous to that obtained in Sec. III for a scalar field. In particular, there are three kinds of modes, excited along the orbifold only, along $\Sigma$ only or along both, as (24) shows. The masses $m_{\Sigma}$ of the first graviton KK modes along $\Sigma$ are of order $1 / R$. However, the modes winding along the orbifold only (the $\Sigma$ zero mode) have masses given by $m_{\text {orb }} \sim a k$, as in the RS model (the curvature scale times the redshift factor). In the approximation of everywhere small $\Sigma$ that we are considering, $k R_{ \pm} \ll 1$, this means that these modes are a factor $a$ lighter than the modes propagating along $\Sigma$.

This allows us to assume the SUSY breaking scale $\eta_{\mathrm{SUSY}}$ and the cutoff $M$ are such that $k \sim T e V$, obtaining quite small masses for the graviton orbifold KK modes $m_{\mathrm{orb}} \sim a T e V$. Below, we show that such a small value does not conflict with observations, since the coupling of these modes to matter is very suppressed. This is consistent with the assumption made above that in the higher dimensional theory the masses of matter fields are near the cutoff $M$, since they are redshifted to $a M \sim T e V$, which compatible with the electroweak scale. Also, since we are considering the limit of everywhere small internal space $k R_{ \pm} \lesssim 1$, setting $k \sim T e V$ implies that the masses of matter KK fields is large enough, $1 / R \gtrsim T e V$.

Thus, from the point of view of the 4 dimensional effective theory, KK modes from the matter fields appear at $1 / R \sim T e V$. Since the curvature scale $k$ of the bulk is close to $1 / R$, this coincides with the scale where gravity becomes higher dimensional.

In summary, we are lead to consider distribution of scales illustrated in Fig. 2. We set the cutoff $M$ and the SUSY breaking scale $\eta_{\mathrm{SUSY}} \lesssim M$ such that the curvature scale of the bulk is $k \sim T e V$. We assume that some mechanism

\footnotetext{
${ }^{9}$ This means that in a certain range of energy the model is effectively 5 dimensional. In appendix B, we derive the form of the dimensionally reduced theory down to 5 dimensions.
} 
can stabilize $R_{-}$near the fundamental length $1 / M$ and $R_{+} \sim 1 / k$. As a consequence, the masses of the graviton KK modes along the orbifold are $m_{\text {orb }} \sim a T e V$, and for the modes along $\Sigma$ are $m_{\Sigma} \sim T e V .10$

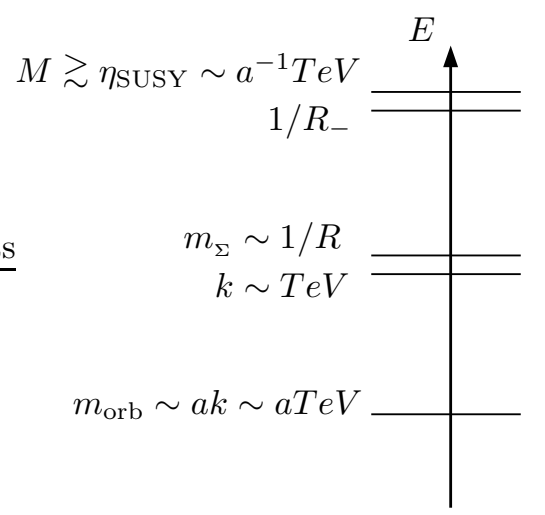

FIG. 2. The mass $m_{\text {orb }}$ of the first KK excitations along the orbifold is much smaller than the mass $m_{\Sigma}$ of the modes excited along the internal manifold. There is essentially the same hierarchy between the fundamental cutoff $M$ and the scales that determine the solution, $k$ and $R$.

It remains to be seen that, indeed, the graviton KK modes along the orbifold are unobservable, in spite of their relatively small masses $m_{\text {orb }} \sim a k$. We see from Eqs. $(25,26)$ that the (unnormalized) wave function of the KK modes grows exponentially as $e^{D k|y| / 2}$, signaling that the more warped the extra dimensions are, the more localized on the negative tension brane these modes are. This implies [30] that the coupling of these graviton KK modes is amplified with respect to that of the zero mode $\left(\sim 1 / m_{P}\right)$ by a factor $a^{-D / 4}=h^{-D / 2(D-1)}$. Then, they are much more weakly coupled to matter $\sim 1 /\left(10^{8} \mathrm{TeV}\right)$ than in the RS model $(\sim 1 / \mathrm{TeV})$. Thus, in spite of their relatively small mass, these KK gravitons cannot be seen individually in accelerators. Moreover, since they are associated with only one off-the-brane dimension, they do not have as large a multiplicity as in the usual large volume mechanism [3], and so they do not significantly cool stars. The total rate of emission of any of such gravitons at a given energy $E<T e V$ can be estimated as the coupling squared times the number of states with masses lighter than $E$ [3,42],

$$
\left(\frac{1}{a^{D / 4} m_{P}}\right)^{2} \frac{E}{m_{\mathrm{orb}}} \sim h^{(D-4) /(D-1)} \frac{E}{T e V} \frac{1}{T e V^{2}},
$$

which is very small for the energies available inside stars.

\section{STABILIZATION}

The result we have obtained so far $(61)$ is a potential $V\left(R_{ \pm}\right)$for the two moduli describing the background. Using $r_{ \pm} \equiv k R_{ \pm}$, it can be cast as

$$
V\left(r_{ \pm}\right)=-\frac{1}{32 \pi^{2} R^{4}}\left[V_{+}\left(r_{+}\right)+V_{-}\left(r_{-}\right)+v\left(r_{+}, r_{-}\right)\right]
$$

\footnotetext{
${ }^{10}$ Another interesting possibility consists of setting $m_{\text {orb }} \sim T e V$ so that $k \sim a^{-1} T e V$ and $M \sim a^{-2} T e V$. This could be realized in a scenario with the SUSY breaking scale $\eta_{\text {SUSY }} \sim 1 / R \sim k$ and the masses of particles of order $k$, from the $(D+1)$ viewpoint. In this scenario, the EW/Planck hierarchy is given by $h^{2} \sim a^{2} k^{2} / m_{P}^{2}$. If the moduli are stabilized so that $R+\lesssim 1 / k$ and $R_{-} \gtrsim 1 / M$, then $h \sim(k / M)^{D+1}$, thus needing less separation between $\eta_{\text {SUSY }}$ and $M$ in order to explain same hierarchy $h$. Moreover, one can see that the potential (61) generates masses for the moduli larger than in the scenario presented so far. However, the bulk cosmological constant $\Lambda$ would be much larger than $\eta_{\mathrm{SUSY}}^{D+1}$.
} 
where $v(R, r)$ contains the 'non-local' part, and

$$
V_{ \pm}\left(r_{ \pm}\right)=\sum_{j=-1}^{\infty}(\mp 1)^{j}\left\{\gamma_{j} r_{ \pm}^{j}+\left(\beta_{j}-g_{0} d_{4} \delta_{j, 4}\right) r_{ \pm}^{j} \ln r_{ \pm}^{2}-\alpha_{j}^{ \pm} r_{ \pm}^{j}\right\}
$$

Here, the coefficients $\alpha_{j}^{ \pm}$are understood to be finite renormalization constants, and are nonzero when the corresponding logarithmic term is nonzero. This is dictated by $\beta_{j}$ being zero or not (i.e., whether or not such a term is divergent), with the sole exception of $j=4$. If $\beta_{4}=0$ and the laplacian $P_{\Sigma}$ (see Eq. (20)) has one zero eigenvalue, $g_{0}=1$, the logarithmic terms corresponding to $j=4$ are not associated to any divergence of the effective action, and $\alpha_{4}^{ \pm}=0$. This situation arises, for example, when $\Sigma$ is a torus.

Note that the sum goes from -1 to $\infty$ and we recall that from Eq. (55), all the $\beta_{j}$ with $j>4+D_{2}$ vanish identically. Thus, the term $\beta_{j} r_{-}^{j}$ appears with $j$ running from -1 to $D_{2}+4$, and the same holds for the terms with $\alpha_{j}^{ \pm}$(there are a finite number of divergent terms).

One interesting feature of the effective potential (66) in both cases with $D_{2}$ even and odd is that the two leading terms in the small $r_{ \pm}$limit (corresponding to $j=-1,0$ ) do not depend on the mass $m$ nor the non-minimal coupling constant $\xi$. This means that if we consider equal number of fermionic and bosonic degrees of freedom, these terms cancel identically even with non supersymmetric masses. From now on, we will focus on this case, one motivation being that the models considered here arise mainly in string theories, and the field content of the effective theories indeed contain equal number of bosonic and fermionic degrees of freedom. The only change is that the sum in Eq. (67) will begin at $j=1$ instead of $j=-1$. As mentioned above, the effective potential contains a finite number of renormalization parameters $\alpha_{j}^{ \pm}$. Their values are not computable from our effective theory. Rather, we shall fix them by requiring some renormalization conditions, which determine the values for the moduli as well. Since the moduli must be stabilized, we demand

$$
\partial_{r_{+}} V\left(r_{ \pm}\right)=\partial_{r_{-}} V\left(r_{ \pm}\right)=0
$$

and in order to match the observed value of the effective four dimensional cosmological constant, we shall impose

$$
\left.V\left(r_{ \pm}\right)\right|_{\min } \simeq 10^{-122} m_{P}^{4}
$$

We are interested in the limit when the size of $\Sigma$ is everywhere smaller than the orbifold size, $r_{+} \lesssim 1$ and $r_{-} \ll 1$. One can show ${ }^{11}$ that in this limit the non-local term $v\left(r_{ \pm}\right)$is exponentially suppressed, and we can approximate the potential by the 'local' terms $V_{ \pm}\left(r_{ \pm}\right)$. Moreover, since we consider only the positive powers of $r_{ \pm}$in $V_{ \pm}$, the potential at the minimum is dominated by $r_{+}$. Then, conditions (68) and (69) reduce to

$$
V_{+}^{\prime}\left(r_{+}\right)=V_{-}^{\prime}\left(r_{-}\right)=0, \quad \text { and }\left.\quad R^{-4} V_{+}\left(r_{+}\right)\right|_{\min } \simeq 10^{-122} m_{P}^{4} .
$$

To investigate whether this potential can stabilize the moduli, we consider separately the cases with flat and curved $\Sigma$.

\footnotetext{
${ }^{11}$ For instance, consider a the six dimensional example, with $\Sigma=S^{1}$. As described in more detail in Subsect. VII A, the generalized zeta function is related to the Riemann zeta function. In this case the we can easily work out the asymptotic behaviour of the nonlocal contribution due to the mixed KK states $\mathcal{V}_{l}\left(a, R_{-}\right)$defined in $(57)$. If we keep the first term in the asymptotic expansion of the Bessel functions (46)

$$
\begin{aligned}
\mathcal{V}_{l}\left(a, R_{-}\right) & \sim \int_{1}^{\infty} d z\left(z^{3}-z\right) \ln \left(1-e^{-2(1-a) y_{l} z}\right) \\
& =-\frac{1}{8} \frac{1}{y_{l}^{4}(1-a)^{4}}\left\{4(1-a)^{2} y_{l}^{2} \operatorname{Li}_{3}\left(e^{-2(1-a) y_{l}}\right)+6(1-a) y_{l} \operatorname{Li}_{4}\left(e^{-2(1-a) y_{l}}\right)+3 \operatorname{Li}_{5}\left(e^{-2(1-a) y_{l}}\right)\right\} .
\end{aligned}
$$
}

Taking only the first term in the series of the poly-logarithms for small arguments $\operatorname{Li}_{n}(z) \approx z$, recalling that $y_{l}=\hat{\lambda}_{l} / r_{-}$with $\hat{\lambda}_{l}=l, g_{l}=2$ and summing over $l=1,2, \ldots$, we find to leading order

$$
\sum_{l=1}^{\infty} g_{l} \hat{\lambda}_{l}^{4} \mathcal{V}_{l}\left(a, R_{-}\right) \sim-\frac{1}{(1-a)^{2}} r_{-}^{2} e^{-2(1-a) / r_{-}}
$$

Thus, this contribution is safely negligible in the limit of small internal space size $r_{-} \ll 1$. 


\section{A. Flat $\Sigma$}

This case corresponds to a toroidal compactification of a $4+D_{2}+1$ dimensional RS model (with two codimension one branes). In this case, all the divergences have the same form, because all geometric invariants are constant and thus proportional to the brane tensions. Thus, there will appear a logarithmic term in the $\left(4+D_{2}\right)$-th power of $r_{ \pm}$. As can also be derived from Eqns. (61), (55) and (56), setting $C_{j}=0$ for all $j \neq 0$, and $g_{0}=1$, there is another logarithmic term corresponding to $j=4$.

Thus, the expression for the potential reduces to

$$
\begin{aligned}
V_{ \pm}\left(r_{ \pm}\right) & \approx\left\{\mp \gamma_{1} r_{ \pm}+\gamma_{2} r_{ \pm}^{2} \mp \gamma_{3} r_{ \pm}^{3}+\left(\gamma_{4}-d_{4} \ln r_{ \pm}^{2}\right) r_{ \pm}^{4}+\ldots\right. \\
& \left.+(\mp 1)^{4+D_{2}} \beta_{4+D_{2}} r_{ \pm}^{4+D_{2}} \ln r_{ \pm}^{2}-\alpha_{4+D_{2}}^{ \pm} r_{ \pm}^{4+D_{2}}+\ldots\right\}
\end{aligned}
$$

To illustrate better how the stabilization mechanism works in these cases, we shall discuss in more detail the six dimensional example with $\Sigma=S^{1}$.

The laplacian (20) on this flat manifold is $P_{\Sigma}=\partial_{\theta}^{2} / R^{2}$, and its generalized zeta function $(36$,A2) is related to the Riemann zeta function through

$$
\zeta\left(s \mid \partial_{\theta}^{2}\right)=2 \zeta_{R}(2 s)
$$

The pole structure of $\zeta_{R}(2 s)$ is easily found and one immediately identifies

$$
\beta_{j}= \begin{cases}-4 d_{5} / 3 & \text { for } j=5 \\ 0 & \text { otherwise }\end{cases}
$$

and

$$
\gamma_{j}= \begin{cases}-2 / 945 & \text { for } j=-1, \\ 3 \zeta_{R}^{\prime}(-4) & \text { for } j=0, \\ 4 \zeta_{R}^{\prime}(-2) d_{2} & \text { for } j=2, \\ -d_{4} & \text { for } j=4, \\ -\frac{8 d_{j} \zeta_{R}(j-4)}{(j-4)(j-2)} & \text { otherwise. }\end{cases}
$$

From (71), the potential is of the form

$$
V_{ \pm}\left(r_{ \pm}\right) \approx\left\{\mp \gamma_{1} r_{ \pm}+\gamma_{2} r_{ \pm}^{2} \mp \gamma_{3} r_{ \pm}^{3}+\left(\gamma_{4}-d_{4} \ln r_{ \pm}^{2}\right) r_{ \pm}^{4} \mp \beta_{5} r_{ \pm}^{5} \ln r_{ \pm}^{2}-\alpha_{5}^{ \pm} r_{ \pm}^{5}+\ldots\right\}
$$

As we mentioned above, the renormalization constants $\alpha_{5}^{ \pm}$arise from a finite renormalization $\delta \tau_{ \pm}$of the brane tensions,

$$
\delta \tau_{ \pm} \int d^{5} x \sqrt{g_{(5) \pm}}=\frac{2 \pi}{R^{4}} \int d^{4} x \delta \tau_{ \pm} R_{ \pm}^{5},
$$

so that $\alpha_{5}^{ \pm}=2 \pi \delta \tau_{ \pm} / k^{5}$. The size of $\delta \tau_{ \pm}$is expected to be set by the SUSY breaking scale $\eta_{\mathrm{SUSY}}$ so that $\alpha_{5}^{ \pm}$are large in principle. Then, the main contributions to this potential arise from the fifth and the first powers. The extremum condition for the $r_{-}$modulus can be well approximated by

$$
\delta \tau_{-} \simeq \frac{\gamma_{1}}{10 \pi} \frac{1}{r_{-}^{4}} k^{5}
$$

Setting the natural value $\delta \tau_{-} \sim \eta_{\mathrm{SUSY}}^{5}$, we obtain

$$
r_{-} \sim\left(\frac{M}{\eta_{\mathrm{SUSY}}}\right)^{1 / 2} \frac{k}{M}
$$

so that indeed $R_{-}$is stabilized just above the fundamental scale $1 / M$ without fine tuning.

As for $r_{+}$, we have two conditions for just one variable, $\delta \tau_{+}$. The idea is to use the renormalization constant $\delta \tau_{+}$ in order to satisfy $\left.V_{+}\right|_{\min } \simeq 0$, and then using this value in $V_{+}^{\prime}=0$, the $r_{+}$is determined. In order to be consistent, 
we should obtain $r_{+} \lesssim 1$. In such a case, we can foresee from Eq. (74) that if $\alpha_{5}^{+}$has to compensate for the potential at the minimum, it has to be of order one. But this means that $\delta \tau_{+}$is fine tuned to a value $\sim k^{5}$ instead of $\eta_{\mathrm{SUSY}}^{5}$.

Imposing explicitly these conditions, we obtain

$$
\delta \tau_{+} \sim \frac{\gamma_{1}}{6 \pi} \frac{1}{r_{+}^{4}} k^{5} \sim k^{5}
$$

and

$$
r_{+} \simeq \frac{4 \gamma_{1}}{3 \gamma_{2}} \sim 1
$$

We can easily check that for a twisted field this ratio is $\simeq 0.6$, in agreement with the assumption we made above. For the untwisted case, this ratio depends on the boundary and bulk masses, so it can be made small generically. In conclusion, besides the fine tuning needed in order to match the four dimensional cosmological constant, no tuning is needed for the Planck/EW hierarchy in this case.

A simple computation gives the mass that this potential induces for the canonical moduli $\Phi$ and $\Psi$ of section II B

$$
\begin{aligned}
& m_{\Phi}^{2} \simeq-\frac{\gamma_{1}}{24 \pi^{2}} \frac{k}{R^{3} m_{P}^{2}} \sim-\gamma_{1}(h k)^{2} \sim(1 / m m)^{2} \\
& m_{\Psi}^{2} \simeq-\frac{\gamma_{1}}{192 \pi^{2}} a^{-2} \frac{k}{R^{3} m_{P}^{2}} \sim-\gamma_{1}(a T e V)^{2},
\end{aligned}
$$

where we used $1 / R \sim k \sim T e V$ and Eq. (65). The mass of $\Phi$ is of the order of the inverse millimeter, which is large enough in order not to cause deviations from Newton's law at short distances. Since, as shown in section II B, the coupling of $\Phi$ to matter is suppressed by a Planckian factor, its effects in accelerators are negligible as well. On the other hand, the mass for the modulus $\Psi$ is of $10 \mathrm{KeV}$ size. From (15), its coupling to matter is suppressed as $1 /\left(10^{4} \mathrm{TeV}\right)$.

Let us briefly discuss the stabilization when we consider a higher dimensional flat $\Sigma$. As mentioned above, with more flat dimensions, the renormalization constants related to the brane tensions $\alpha_{D}^{ \pm}$appear with higher powers of $r_{ \pm}$. The only change with respect to the case above is that the condition $V_{-}^{\prime}=0$ now reads

$$
\delta \tau_{-} \sim \frac{1}{r_{-}^{D-1}} k^{D}
$$

and assuming a natural value for $\delta \tau_{-}$given by $\eta_{\mathrm{SUSY}}^{D}$, we obtain again $r_{-} \sim(k / M)\left(M / \eta_{\mathrm{SUSY}}\right)^{1 / 2}$. Thus, for any dimension $D$ the modulus $R_{-}$is stabilized without fine tuning near $1 / M$.

As for the modulus $R_{+}$, we expect the potential (71) to stabilize it near $k$ once the fine tuning of $\delta \tau_{+} \sim k^{D}$ needed for the cosmological constant is performed.

We can compute the masses for the moduli for an arbitrary number of flat internal dimensions. We find that the mass for the $\Phi$ is always millimetric, whereas $m_{\Psi} \sim a T e V$ increases with $D_{2}$, ranging from $10 \mathrm{KeV}$ for $D_{2}=1$ to $100 \mathrm{MeV}$ for $D_{2}=6$. The coupling of $\Psi$ to matter, of strength (see Eq. (15))

$$
1 /\left(h^{-1 /(D-1)} \mathrm{TeV}\right),
$$

is comprised between $\sim 1 /\left(10^{4} \mathrm{TeV}\right)$ for $D_{2}=1$ and $\sim 1 /(100 \mathrm{TeV})$ for large $D_{2}$. This guarantees that it hasn't been produced at colliders, or has any effect in star cooling.

\section{B. Curved $\Sigma$}

When $\Sigma$ is not flat, besides the divergences proportional to brane tensions terms (giving rise to the power $r_{ \pm}^{D}$ in $V_{ \pm}$), the potential has more divergences. For instance, there can appear divergences proportional to curvature terms, which give rise to the powers $r_{ \pm}^{D-2}$. Accordingly, terms with fewer powers of $r_{ \pm}$are due to higher powers of the curvature, and in general the effective potential takes the form

$$
V_{ \pm}\left(r_{ \pm}\right)=\sum_{j=1}^{\infty}(\mp 1)^{j}\left\{\gamma_{j} r_{ \pm}^{j}+\left(\beta_{j} r_{ \pm}^{j}-g_{0} d_{4} \delta_{j, 4}\right) \ln r_{ \pm}^{2}\right\}-\sum_{j=1}^{D} \alpha_{j}^{ \pm} r_{ \pm}^{j}
$$


As in the previous case for the brane tensions, the size of the renormalization constants in front of these operators are expected to be of order the cutoff scale $M$ (or $\eta_{\mathrm{SUSY}}$ ). Finite renormalization terms of boundary operators behave as,

$$
M^{j} \int d^{D} x \sqrt{g_{(D) \pm}} \mathcal{R}^{(D-j) / 2}=\frac{1}{R^{4}} \int d^{4} x\left(M R_{ \pm}\right)^{j}=\frac{1}{R^{4}} \int d^{4} x \alpha_{j}^{ \pm} r_{ \pm}^{j}
$$

and we conclude that the dimensionless renormalization constants in (77) are large, $\alpha_{j}^{ \pm} \sim(M / k)^{j} \gg 1$. Thus, these terms are a series in $M R_{ \pm}>1$ rather than in $k R_{ \pm}<1$, the dominant terms being with the highest powers, that is, the brane tension and the curvature terms. As a first approximation, we can neglect the remaining terms, and minimum condition for $R_{-}$is reached naturally for $R_{-} \sim 1 / M$, which is what we need (see Fig. 2).

However, we see that in order to obtain $R_{+} \sim 1 / k$, we need to tune the ratio of $\alpha_{D}$ and $\alpha_{D-2}$. Besides, the tuning corresponding to the cosmological constant is still needed.

In principle, we could consider the case when the heat kernel coefficient $C_{1}\left(P_{\Sigma}\right)$ is zero, which can happen for some value of the non-minimal coupling $\xi$. We see from (55) that in this case there is no divergence in the potential corresponding to the curvature terms. ${ }^{12}$ Then, assuming that the next nonzero coefficient is $C_{2}$, the two powers that dominate the potential are $\left(M R_{ \pm}\right)^{D}$ and $\left(M R_{ \pm}\right)^{D-4}$. However, in order to stabilize $R_{+}$near $1 / k$, again we have to do one fine tuning. We can say that in general, the presence of any other divergence, besides the brane tension, spoils the efficiency of the potential in stabilizing the moduli at well separated scales.

We conclude that, for curved $\Sigma$ the potential can naturally stabilize the moduli but without a large hierarchy.

\section{CONCLUSIONS}

In this article we have investigated the role of quantum effects arising from bulk scalar fields in higher dimensional brane models. Specifically, we have considered a class of warped brane models whose topology is $\mathcal{M} \times \Sigma \times S^{1} / Z_{2}$, where $\Sigma$ is a $D_{2}$ dimensional one-parameter compact manifold, $\mathcal{M}$ is the four dimensional Minkowski space and both $\mathcal{M}$ and $\Sigma$ directions are warped as in the Randall Sundrum model, with two branes of codimension one sitting at the orbifold fixed points. Aside from the usual negative cosmological constant, a bulk sigma model scalar field theory is used as the source of gravity in the cases of a curved internal manifold $\Sigma$. We have identified the relevant moduli fields characterizing the background, and found the classical action in the moduli approximation (as well as the coupling of the moduli to matter fields sitting on the branes).

We have computed the contribution to the one-loop effective action from generic bulk scalar fields at lowest order (i.e. the Casimir energy). The computation, similar to the one for the RS model, is technically more complex since there are KK modes propagating along $\Sigma$, resulting in a dependence of the Kaluza-Klein masses on the eigenvalues of the Klein-Gordon operator on $\Sigma$. However, for the specific choice of space-time we made, where the warp factors for $\Sigma$ and for the Minkowski factor $\mathcal{M}$ are the same, the physical KK masses split as in the usual factorisable geometries. Using the Mittag-Leffler expansion for the generalized $\zeta$-function we were able to express the Casimir energy in terms of heat-kernel coefficients of the internal space $\Sigma$, so that the presence of each divergence is dictated by a certain heat-kernel coefficient. This simplifies the renormalization of the result. An interesting nontrivial check of our result is the fact that the RS divergence (which is lower dimensional in this model and which appears as the contribution of the $\Sigma$ zero mode) cancels out in the final result, as it should, once all contributions are added. We renormalized the effective potential by subtracting suitable counter-terms proportional to a number of boundary or bulk local operators. Since we work in dimensional regularization, the subtraction is performed in the regularized space, with $(4-2 \epsilon)+D_{2}+1$ dimensions. As a result, there is a mismatch in the powers of the moduli appearing in the divergent terms, and a number of logarithmic terms (in the moduli) appear in the renormalized expression for the effective potential.

As an application, we proposed a scenario where SUSY is broken at a scale just below the fundamental cutoff $M$. This makes the curvature scale of the background to be several orders of magnitude below $M$. As a result, a large hierarchy is generated by a combination of redshift [6] and a large volume effects [3]. The key point for the latter to be efficient (in spite of having codimension one branes) is that the size of the internal manifold $\Sigma$ (present in the bulk and on the brane) grows as one moves away from the $\mathrm{TeV}$ brane, where matter lives. Therefore, this behaves effectively as a brane with a small $\Sigma$ extra space, attached to which there is a large $\Sigma$ space where only gravity propagates.

\footnotetext{
${ }^{12}$ The same thing cannot happen for the brane tension terms, since the corresponding coefficient is $C_{0}\left(P_{\Sigma}\right)=1$ always.
} 
As for the stabilization, we find that, generically, the potential induced by bulk fields can generate sizeable masses for the moduli compatible with a large hierarchy with no need of fine tuning if $\Sigma$ is flat. If it is curved, the effective potential can naturally stabilize the moduli but without a large hierarchy.

In the model we have considered, the size of the internal space $\Sigma$ is everywhere small compared with the size of the orbifold. Therefore, there is a range of energy scales where the model is effectively five dimensional (this feature is in common with the Hořava-Witten model $[4,5]$ ). From the five-dimensional point of view, the model contains a dilaton field in the bulk, which causes a power-law warp factor in the Einstein frame, $a(y) \propto y^{q}$, where $y$ is the proper distance along the extra dimension. The power $q$ is related to the number of additional dimensions through $q=\left(D_{2}+3\right) / D_{2}$ [14], which leads to $1<q \leq 4$. Five dimensional models with power-law warp factors were investigated in [14], where it was argued that the counterterms at the orbifold fixed points can naturally stabilize the moduli corresponding to the positions of the branes. However, a large hierarchy was not expected unless the power is substantially large, $q \gtrsim 10$. This conclusion is consistent with the results of the present paper, which correspond to relatively small $q$. In this case, the large hierarchy can only be stabilized naturally if the internal space is flat. This case is special because the only possible counterterms are renormalizations of the higher dimensional brane tensions.

The above arguments suggest that a large hierarchy may be obtained by considering more general warped models, where a larger power exponent $q$ is obtained after reducing to five dimensions. In such cases, the stabilization of a hierarchy without fine tuning is expected even if the internal manifold $\Sigma$ is curved and all sorts of counterterms are present. As a continuation of the work presented here, we are currently considering the case in which the warp factor along $\Sigma$ is constant [25,29]. We hope to report on this shortly [43].

\section{ACKNOWLEDGMENTS}

J.G. and O.P. are grateful to Alex Pomarol for very useful discussions. A.F. acknowledges discussions with Y. Himemoto, M. Sasaki and W. Naylor and the members of the Department of Earth and Space Science at Osaka University for the kind hospitality. A.F. is supported by the European Community via the award of a Marie Curie Fellowship. We acknowledge support by the DURSI Research Project 2001SGR00188, by CICYT under Research Projects AEN99-0766, and the MCyT and FEDER under projects FPA 2002-3598, FAP 2002-00748 and FAP 200200648, and HPRN-CT-2000-00131. T.T. is supported by a Monbukagakusho Grant-in-Aid No. 14740165. To complete this work, the discussion during and after the YITP workshop YIYP-W-01-15 on "Braneworld - Dynamics of spacetime boundary" was useful.

\section{APPENDIX A: MITTAG-LEFFLER EXPANSION}

In the present appendix we prove Eq. (38). It is well known that for a strictly positive definite laplacian $P$ with eigenvalues $\lambda_{P}$, the associated zeta function $\zeta(s \mid P)=\sum \lambda_{P}^{-s}$ admits a Mittag-Leffler expansion of the form

$$
\zeta(s \mid P)=\frac{1}{\Gamma(s)}\left\{\sum_{p=0}^{\infty} \frac{C_{p}(P)}{s-D_{2} / 2+p}+f(s \mid P)\right\}
$$

where $C_{p}(P)$ are the Seeley-DeWitt coefficients related to $P$, with $p \in \mathbb{N} / 2$ and the function $f(s \mid P)$ is analytic for all finite $s$. This is a very useful relation since it neatly shows the pole structure of the zeta function, in terms of geometrical invariants.

We need to generalize this equation to operators with one zero eigenvalue $\left(g_{0}=1\right)$. Consider a positive semidefinite differential operator $P_{\Sigma}$ with eigenvalues $\lambda_{l}^{2}$ and assume that there is one zero eigenvalue. As usual in these cases, one defines the generalized $\zeta$ function excluding this eigenvalue (see Eq. (36)),

$$
\zeta(s)=\sum_{l=1}^{\infty} \hat{\lambda}_{l}^{-2 s} .
$$

Our main task is to express $\zeta(s)$ in terms of geometrical quantities in the form

$$
\zeta(s)=\frac{1}{\Gamma(s)}\left\{\sum_{p=0}^{\infty} \frac{\tilde{C}_{p}}{s-D_{2} / 2+p}+f(s)\right\},
$$


with $p \in \mathbb{N} / 2$, for some $\tilde{C}_{p}$ related to the Seeley-DeWitt coefficients of $P_{\Sigma}$. First of all, let us introduce the regulated zeta function associated to the operator ${ }^{13} P_{\Sigma}^{\mu} \equiv P_{\Sigma}+\mu^{2} / R^{2}$,

$$
\zeta_{\mu}(s)=\sum_{l=0}^{\infty}\left(\hat{\lambda}_{l}^{2}+\mu^{2}\right)^{-s} .
$$

Now it is trivial to express the function $\zeta(s)$ in terms of $\zeta_{\mu}(s)$,

$$
\zeta(s)=\lim _{\mu \rightarrow 0}\left(\zeta_{\mu}(s)-\mu^{-2 s}\right),
$$

and it is obvious that, understood as this limit, $\zeta(s)$ is infrared finite for any $s$, even though $\zeta_{\mu}(s)$ is only IR finite for $\operatorname{Re}(s) \leq 0$. By construction, $P_{\Sigma}^{\mu}$ is strictly positive definite, so $\zeta_{\mu}(s)$ admits the expansion

$$
\zeta_{\mu}(s)=\frac{1}{\Gamma(s)}\left\{\sum_{p=0}^{\infty} \frac{C_{p}(\mu)}{s-D_{2} / 2+p}+g(\mu, s)\right\},
$$

where the function $g(\mu, s)$ is analytic and the Seeley-DeWitt coefficients $C_{p}(\mu)$ depend polynomially on $\mu$.

From Eqns. $(\mathrm{A} 5, \mathrm{~A} 6)$ it follows that

$$
\zeta(s)=\frac{1}{\Gamma(s)} \lim _{\mu \rightarrow 0}\left\{\sum_{p=0}^{\infty} \frac{C_{p}(\mu)}{s-D_{2} / 2+p}+g(\mu, s)-\Gamma(s) \mu^{-2 s}\right\} .
$$

The next step is to isolate the poles from the last term in the previous formula. We do this expanding $\Gamma(s)$ as

$$
\Gamma(s)=\Gamma(1, s)+\sum_{p=0}^{\infty} \frac{b_{2 p}}{(s+2 p)},
$$

where $\Gamma(z, s)$ is the incomplete gamma function, and the coefficients of the expansion are given by $b_{2 p}=(-1)^{2 p} / p$ ! Using (A8) we have:

$$
\Gamma(s) \mu^{-2 s}=\sum_{p=0}^{\infty} b_{2 p} \frac{\mu^{4 p}}{s+2 p}+\sum_{p=0}^{\infty} h_{p}(\mu, s)+\Gamma(1, s) \mu^{-2 s},
$$

where we have defined

$$
h_{p}(\mu, s)=b_{2 p} \frac{\mu^{-2 s}-\mu^{4 p}}{s+2 p} .
$$

Eq. (A9) allows us to write

$$
\zeta(s)=\frac{1}{\Gamma(s)} \lim _{\mu \rightarrow 0}\left\{\sum_{p=0}^{\infty} \frac{\tilde{C}_{p}(\mu)}{s-D_{2} / 2+p}+f(\mu, s)\right\}
$$

where

$$
f(\mu, s)=g(\mu, s)-\sum_{p=0}^{\infty} h_{p}(\mu, s)-\Gamma(1, s) \mu^{-2 s},
$$

and the modified coefficients $\tilde{C}_{p}(\mu)$ are then given by

$$
\tilde{C}_{D_{2} / 2+2 p}(\mu)=C_{D_{2} / 2+2 p}(\mu)-b_{2 p} \mu^{4 p}
$$

\footnotetext{
${ }^{13}$ Note that this mass is fictitious and has nothing to neither with the physical bulk mass $m$ (16) nor with the renormalization scale.
} 
We note that the only coefficients which are modified are $C_{D_{2} / 2}, C_{D_{2} / 2+1}, C_{D_{2} / 2+2}, \ldots$ Taking the limit $\mu \rightarrow 0$, we obtain the main result of this appendix

$$
\zeta(s)=\frac{1}{\Gamma(s)}\left\{\sum_{p=0}^{\infty} \frac{\tilde{C}_{p}}{s-D_{2} / 2+p}+f(s)\right\},
$$

with

$$
\tilde{C}_{p} \equiv \lim _{\mu \rightarrow 0} \tilde{C}_{p}(\mu)=C_{p}(0)-\delta_{2 p, D_{2}},
$$

where $\delta_{p, p^{\prime}}$ is the Kronecker delta, the limit of $C_{p}(\mu)$ can be taken because they are polynomials in $\mu$, and the function $f(s)=f(0, s)$ is analytic and finite by construction, although it can be explicitly checked from (A12).

In conclusion, the result (A14,A15) implies that for a laplacian with one zero eigenvalue, there also exists a MittagLeffer-like expansion for the 'primed' zeta function (A2,36), changing only the Seeley-DeWitt coefficient $C_{D_{2} / 2}$ by $C_{D_{2} / 2}-1$. That is why we can consider Eq. (A6) valid in general (with either $g_{0}=1$ or 0 ), replacing $C_{D_{2} / 2}$ by $C_{D_{2} / 2}-g_{0}$.

It is now easy to expand around $s=p$ and a simple calculation gives

$$
\left.\Gamma(s) \zeta(s)\right|_{s=p}=\frac{\tilde{C}_{D_{2} / 2-p}}{s-p}+\Omega_{p}+\mathcal{O}\left((s-p)^{2}\right),
$$

with

$$
\Omega_{p} \equiv \sum_{p^{\prime} \neq D_{2} / 2-p} \frac{\tilde{C}_{p^{\prime}}}{p+p^{\prime}-D_{2} / 2}+f(p)
$$

\section{APPENDIX B: $(D+1) \rightarrow 5$ REDUCTION}

In section VI, we have argued that there exists a range of energies where the theory is effectively 5 dimensional, as illustrated in Fig. 2.

In this appendix we show the dimensional reduction procedure from $(D+1)$ dimensions down to 5 dimensions, which allows contact with the language of [14].

The reduction from the higher dimensional theory (2) to 5 dimensions is performed by the compactification on the internal manifold $\Sigma$. This amounts to keeping only the $\Sigma$-zero modes of the fields defined in $(D+1)$ dimensions.

In this section we denote collectively the four dimensional Minkowski coordinates $x^{\mu}$ and the orbifold $x^{5}$ by $x^{a}$.

For the sake of simplicity, we shall consider only the breathing mode of $\Sigma$ in the internal components of the metric. As well, we shall freeze the $\{a, i\}$ components (the graviphotons) to zero.

Thus, the ansatz for the metric that we shall adopt depends on the internal coordinates $X^{i}$ only through the background geometry on $\Sigma$, and on $x^{a}$ through the five dimensional graviton $g_{a b}^{(5)}$, and a dilaton $\sigma$,

$$
d s^{2}=g_{a b}^{(5)}\left(x^{c}\right) d x^{a} d x^{b}+R^{2} e^{2 \sigma\left(x^{c}\right)} \gamma_{i j} d X^{i} d X^{j} .
$$

As for the sigma model scalars, we shall also freeze them to their value in the background, $\phi^{a}=\phi^{a}\left(X^{i}\right)$.

The action (2) corresponding to this ansatz is

$$
\begin{aligned}
S_{5}=-v_{\Sigma} R^{D_{2}} & {\left[\int d^{5} x \sqrt{g_{(5)}} e^{D_{2} \sigma}\left\{M^{D-1}\left(\mathcal{R}_{(5)}-D_{2}\left(D_{2}-1\right)(\partial \sigma)_{(5)}^{2}\right)+\Lambda\right\}\right.} \\
+ & \left.\int d^{4} x \sqrt{g_{(5)+}} e^{D_{2} \sigma} \tau_{+}+\int d^{4} x \sqrt{g_{(5)-}} e^{D_{2} \sigma} \tau_{-}\right]
\end{aligned}
$$

where $g_{\mu \nu}^{(5) \pm}$ denote the metrics on the branes induced by $g_{a b}^{(5)}$ and we have performed the $X$ integration. We can rewrite this action in the (5 dimensional) Einstein frame, given by $g_{a b}^{E}=e^{2 D_{2} \sigma / 3} g_{a b}^{(5)}$,

$$
\begin{aligned}
S_{5}= & -M_{5}^{3} \int d^{5} x \sqrt{g_{E}}\left\{\mathcal{R}_{E}+\frac{1}{2}(\partial \phi)_{E}^{2}+\Lambda_{5} e^{c \phi}\right\} \\
& -\int d^{4} x \sqrt{g_{E+}} \tau_{5+} e^{c \phi / 2}-\int d^{4} x \sqrt{g_{E-}} \tau_{5-} e^{c \phi / 2}
\end{aligned}
$$


where

$$
c^{2}=\frac{2}{3} \frac{D_{2}}{D_{2}+3},
$$

the canonical scalar field is $\phi=-\left(2 D_{2} / 3 c\right) \sigma, g_{\mu \nu}^{E \pm}$ are the metrics on the branes induced by $g_{a b}^{E}$, the 5 dimensional Planck mass is given by $M_{5}^{3}=v_{\Sigma} R^{D_{2}} M^{D-1}, \Lambda_{5}=M^{1-D} \Lambda$ and $\tau_{5 \pm}=v_{\Sigma} R^{D_{2}} \tau_{ \pm}$.

The action (B3) coincides with the 5 dimensional scalar-tensor model considered in [14]. It was found there that this model has a solution with a power-law warp factor of the form

$$
\begin{aligned}
d s_{E}^{2} & =a_{E}^{2}(z)\left(d z^{2}+\eta_{\mu \nu} d x^{\mu} d x^{\nu}\right), \\
\phi_{0}(z) & =-\sqrt{6 \beta(\beta+1)} \ln \left(z / z_{0}\right) \quad \text { with } \quad a_{E}(z)=\left(z / z_{0}\right)^{\beta}
\end{aligned}
$$

with $\beta=2 /\left(3 c^{2}-2\right)=-\left(D_{2}+3\right) / 3 .{ }^{14}$

The brane operators induced by quantum effects on this background are given by positive powers of the extrinsic curvature scale (see e.g. [14]) $\mathcal{K}_{E \pm}=\beta / z_{ \pm} a_{E \pm}=\beta z_{ \pm}^{-(\beta+1)}$,

$$
\int d^{4} x \sqrt{g_{E}} \mathcal{K}_{E \pm}^{n}=\int d^{4} x\left(\frac{z_{ \pm}}{z_{0}}\right)^{(4-n) \beta} \frac{1}{z_{ \pm}^{n}} \propto \int d^{4} x r_{ \pm}^{4+(4 / 3) D_{2}-(n / 3) D_{2}},
$$

where $n=1,2,3 \ldots$, we used that the conformal coordinate $z=e^{k y}$ is the same in 5 and in $(D+1)$ dimensions, and $a_{ \pm}=1 / k z_{ \pm}$. On the other hand, we have seen in section $\mathrm{V}$ that the operators generated by the effective potential due to bulk fields in the model (2) are of the form

$$
\int d^{4} x \sqrt{g_{ \pm}} \mathcal{R}_{ \pm}^{N} \propto \int d^{4} x r_{ \pm}^{4+D_{2}-2 N}
$$

where $\mathcal{R}_{ \pm}$is the intrinsic curvature computed with the induced metrics on each brane, $g_{\mu \nu}^{ \pm}$. Here $N=0,1, \ldots,[(D+$ 1)/2], and [ ] denotes the integer part. Now we can identify that these operators correspond to a number of powers of the extrinsic curvature operator (B6) given by

$$
n=\frac{6}{D_{2}} N+1
$$

We note that all the induced operators can be cast as powers of the extrinsic curvature for $D_{2}=1,2,3$ and 6 only, having in the $D_{2}=6$ case a one-to-one correspondence. For any other value of $D_{2}$, there exist higher dimensional local operators that are not simply powers of $\mathcal{K}_{E \pm}$, but of some power of $e^{\phi}$ in the 5 dimensional effective theory (B3).

As well, Ref. [14] raised the question that the path integral measure of a bulk scalar field in the effective five dimensional theory (B3) quantized on the warped vacuum configuration (B5) is ambiguously defined. The nontrivial profile of the scalar $\phi$ permits to define many different conformal frames, all of them equivalent at the classical level. However, the path integral measure can be defined covariantly with respect to any of them. It turns out that the term proportional to

$$
\frac{\ln z_{+}}{z_{+}^{4}}+\frac{\ln z_{-}}{z_{-}^{4}}
$$

in the potential depends on this choice. Several arguments can be given in favor of possible 'preferred' frames. For instance, with a measure covariant with respect to the 5 dimensional Einstein frame metric $g_{a b}^{E}$, this term is present. But if one chooses covariance with respect to $g_{a b}^{(5)}$, there is no such term. However, in the model presented here, there is no ambiguity in the choice of the measure since in the $D+1$ dimensional theory there is no scalar with nontrivial profile along the orbifold. In the computation presented here, the choice of the measure shows up (see [14]) when we subtract the divergences Eq. (60), covariant precisely with respect to the higher dimensional Einstein frame metric $g_{M N}^{(D+1)}$. As a result, when we take into account both the 5 dimensional modes (the $\Sigma$ KK zero mode) together with the $D+1$ dimensional ones (the KK modes excited along the $\Sigma$ as well), we have found that there is a remaining

\footnotetext{
${ }^{14}$ In terms of the proper coordinate (in the 5 dimensional Einstein frame) $y_{E} \propto z^{\beta+1}, a_{E}\left(y_{E}\right)=\left(y_{E} / y_{0}\right)^{q}$ with $q=2 / 3 c^{2}=$ $\left(D_{2}+3\right) / D_{2}$.
} 
contribution of this form, see Eqns. $(61,55,56)$. Anyhow, it should be noted that these Coleman-Weinberg-like terms do not play a very relevant role in stabilizing of the moduli.

[1] T. Kaluza, Sitzungsber. Preuss. Akad. Wiss. Berlin Math. Phys. Kl. 33 (1921) 966.

[2] O. Klein, Z. Phys. 37 (1926) 895;

O. Klein, Nature (London) 118 (1926) 516.

[3] I. Antoniadis, Phys. Lett. B246 (1990) 377;

N. Arkani-Hamed, S. Dimopoulos, G. Dvali, ibid. B429 263;

I. Antoniadis, N. Arkani-Hamed, S. Dimopoulos, G. Dvali, ibid. B436 257.

[4] P. Horava, E. Witten, Nucl. Phys. B460 (1996) 506;

P. Horava, E. Witten, Nucl. Phys. B475 (1996) 94.

[5] A. Lukas, B.A. Ovrut, K.S. Stelle, D. Waldram, Phys. Rev. D59 (1999) 086001.

[6] L. Randall, R. Sundrum, Phys. Rev. Lett. 83 (1999) 3370.

[7] J. Garriga, T. Tanaka, Phys. Rev. Lett. 84 (1999) 2778.

[8] W. D. Goldberger and M. B. Wise, Phys. Rev. Lett. 83, 4922 (1999).

[9] P. Candelas, S. Weinberg, Nucl. Phys. B237 (1984) 397.

[10] A. Flachi, D.J. Toms, Nucl. Phys. B610 (2001) 144.

[11] A. Flachi, I.G. Moss, D.J. Toms, Phys. Lett. B518 (2001) 153.

[12] A. Flachi, I.G. Moss, D.J. Toms, Phys. Rev. D64 (2001) 105029.

[13] J. Garriga, O. Pujolàs., T. Tanaka, Nucl. Phys. B605 (2001) 192.

[14] J. Garriga, O. Pujolàs., T. Tanaka, Nucl. Phys. B (2002) [arXiv:hep-th/0111277].

[15] D.J. Toms, Phys. Lett. B484 (2000) 149.

[16] W.D. Goldberger, I.Z. Rothstein, Phys. Lett. B491 (2000) 339.

[17] Y. Himemoto, M. Sasaki, Phys. Rev. D63 (2001) 04015.

[18] N. Sago, Y. Himemoto, M. Sasaki, Phys. Rev. D65 (2001) 024014;

S. Kobayashi, K. Koyama, J. Soda, Phys.Lett. B501 (2001) 157.

[19] W. Naylor, M. Sasaki, Phys. Lett. B542 (2002) 289.

[20] S. Nojiri, S. Odintsov, S. Zerbini, Class. Quantum Grav. 17 (2000) 4855.

[21] E. Elizalde, S. Nojiri, S. Odintsov, S. Ogushi, [arXiv:hep-th/0209242].

[22] S. Mukohyama, Phys. Rev. D63 (2001) 044008.

[23] I. Brevik, K. A. Milton, S. Nojiri and S. D. Odintsov, Nucl. Phys. B599 (2001) 305.

[24] J. Garriga and A. Pomarol, arXiv:hep-th/0212227.

[25] R. Gregory, Phys. Rev. Lett. 84 (2000) 2564.

[26] T. Gherghetta, M. Shaposhnikov, Phys. Rev. Lett. 85 (2000) 240.

[27] T. Gherghetta, E. Roessl and M. E. Shaposhnikov, Phys. Lett. B491 (2000) 353.

[28] I. Oda, Phys. Rev. D64 (2001) 026002.

[29] S. Randjbar.Daemi, M. Shaposhnikov, Phys. Lett. B491 (2000) 329.

[30] H. Davoudiasl, J. L. Hewett, T. G. Rizzo, [arXiv:hep-th/0211377].

[31] S. Randjbar.Daemi, M. Shaposhnikov, Phys. Lett. B492 (2000) 361.

[32] J. Khoury, B. A. Ovrut, P. J. Steinhardt and N. Turok, Phys.Rev.D64 (2001) 123522.

[33] D.J. Toms, Phys. Lett. B129 (1983) 31.

[34] S. Blau, M. Visser, A. Wipf, Nucl. Phys. B310 (1988) 163.

[35] E. Elizalde, S. Leseduarte, A. Romeo, J. Phys. A26 (1993) 2409;

S. Leseduarte, A. Romeo, J. Phys. A27 (1994) 2483.

[36] M. Bordag, E. Elizalde, K. Kirsten, J. Math. Phys. 37 (1996) 895;

M. Bordag, J. S. Dowker, K. Kirsten, Comm. Math. Phys. 182 (1996) 371.

[37] K. Kirsten, Spectral Functions in Mathematics and Physics, Chapman \& Hall/CRC, Boca Raton, FL (2001).

[38] R. Contino, P. Creminelli and E. Trincherini, JHEP 0210, 029 (2002) [arXiv:hep-th/0208002].

[39] Z. Chacko, P. J. Fox, A. E. Nelson and N. Weiner, JHEP 0203, 001 (2002).

[40] Z. Chacko and A. E. Nelson, Phys. Rev. D62, 085006 (2000).

[41] T. Multamaki and I. Vilja, Phys. Lett. B545, 389 (2002).

[42] N. Arkani-Hamed, S. Dimopoulos and G. R. Dvali, Phys. Rev. D59 (1999) 086004.

[43] A. Flachi and O. Pujolas, Phys. Rev. D 68, 025023 (2003) [arXiv:hep-th/0304040]. 